\title{
COLOSSES ET PERSPECTIVE \\ DE LA PRISE EN CONSIDÉRATION DE LA PARALLAXE DANS LA STATUAIRE PHARAONIQUE DE GRANDES DIMENSIONS AU NOUVEL EMPIRE
}

\author{
[Planches XVII-XXXIII] \\ PAR \\ DIMITRI LABOURY \\ FNRS - Université de Liège \\ 24/4, quai Sainte-Barbe \\ B-4020 Liège
}

Dans l'étude que j'ai consacrée à la statuaire de Thoutmosis III, j'avais attiré l'attention sur une paire de statues de ce souverain, aujourd'hui conservées au Musée du Caire (CG 594) et à l'entrée du Musée de plein air de Karnak (MPA.T3.st.1), deux sculptures qui, tout en provenant de l'Akh-menou et en s'intégrant très manifestement dans le style qui caractérise toutes les œuvres contemporaines de ce monument, se distinguent néanmoins de ces dernières par un traitement légèrement différent des proportions du visage et de ses éléments constitutifs ${ }^{1}$. En effet, si tous les détails physionomiques caractéristiques de cette phase stylistique du règne de Thoutmosis III y sont bien présents, vu de face, à hauteur des yeux, le visage de ces deux statues adopte une forme plus triangulaire (en triangle sur pointe) que toutes les autres effigies du roi de même provenance ou de même datation, sans compter que les yeux sont un peu plus ouverts, les sourcils plus haut placés sur le front, le nez un peu plus grand, la bouche légèrement étirée vers le bas et les oreilles plus grandes et plus hautes (pl. XVII-XVIII). L'analyse de ces divergences m'avait incité à conclure qu'elles s'expliquaient très probablement par la taille initiale des deux statues en cause, qui devaient à l'origine avoisiner les $280 \mathrm{~cm}$ de haut. On constate effectivement que ces légères différences s'estompent presque totalement, lorsque l'on regarde les œuvres non pas à hauteur de leur visage, — tel que l'on préconise toujours en Égyptologie de photographier une statue en vue de sa publication -, mais au niveau de l'œil du spectateur debout aux pieds du colosse, soit en tenant compte de la hauteur originale de la sculpture (pl. XIX) ${ }^{2}$. D'autres cas de modification induite par

\footnotetext{
${ }^{1}$ Cf. D. Laboury, La statuaire de Thoutmosis III. Essai d'interprétation d'un portrait royal dans son contexte historique (AegLeod 5), 1998, p. 169-175 (catalogue des œuvres) et 520-2 (analyse stylistique).

${ }^{2}$ En réalité, nous allons le voir, la photographie de droite aurait dû idéalement être prise avec un peu plus de recul.
}

Revue d'Égyptologie 59, 181-230. doi: 10.2143/RE.59.0.2036290

Tous droits réservés @ Revue d'Égyptologie, 2008. 
la taille de la statue ayant déjà été signalés ${ }^{3}$, tout incitait donc à penser qu'il s'agit d'un exemple de prise en considération volontaire - puisque présente sur chacune des deux œuvres, de taille identique — de la parallaxe, afin de rectifier la déformation visuelle due aux dimensions colossales des deux statues. Mais une étude spécifique de cette question, qui peut paraître particulièrement étonnante dans le contexte de l'art égyptien, réputé pour son rejet, voire son ignorance, de la perspective, restait évidemment à fournir et c'est l'objet que je me propose de donner au présent article.

J'ai été amené à aborder à nouveau cette question dans le cadre d'une étude systématique que j'ai entreprise depuis plusieurs années sur la statuaire royale du règne d'Amenhotep IV - Akhénaton et dont je prépare la publication prochaine sous la forme d'une monographie ${ }^{4}$. Diverses circonstances m'ont cependant conduit à en détacher en une contribution spécifique l'examen de la question abordée dans cet article.

Dans toute l'histoire de l'art égyptien telle que nous pouvons la retracer ou l'appréhender aujourd'hui, les statues qui présentent la distorsion faciale la plus marquée et la plus impressionnante sont sans conteste les fameux colosses du Gem-pa-Aton d'Amenhotep IV, — futur Akhénaton —, à Karnak. Depuis leur découverte, au milieu des années 19205, ces sculptures sont associées - dans l'esprit des spécialistes, comme dans celui du grand public à l'épisode atoniste comme de véritables icônes, en complément du non moins célèbre buste de Néfertiti, dit de Berlin (ägyptisches Museum 21.300). Ils sont d'ailleurs utilisés dans pratiquement tous les modèles évolutifs de l'histoire de l'art dit amarnien comme les exemples types du style «caricatural» qui aurait caractérisé les débuts de l'ère atoniste ${ }^{6}$. L'étude systématique de tous les vestiges relatifs à l'évolution de la statuaire sous le règne d'Amenhotep IV - Akhénaton conduit cependant à remettre sérieusement en question cette conclusion, aussi hâtive que largement acceptée.

Il faut tout d'abord constater que le faciès particulier de ces statues est absolument constant sur l'ensemble des fragments attestés de la série - en ce compris ceux que l'on a

\footnotetext{
3 Cf. notamment les observations de Betsy M. Bryan sur l'inclinaison des yeux des colosses d'Amenhotep III dans la cour solaire de son temple funéraire au Kôm el-Hettan, observations présentées dans le catalogue de l'exposition Aménophis III, le Pharaon-Soleil (Paris, Grand Palais), 1993, p. 126 et 128.

${ }^{4}$ Ce projet, intitulé «Recherches sur la réforme introduite par Akhénaton dans le système de représentation de l'Égypte antique, ses antécédents, ses prolongements et ses implications pour une théorie générale de l'image pharaonique dans le cadre de la pensée égyptienne», a été déposé comme mon projet de recherche principal auprès du FNRS depuis 1998. Il a pu être mené grâce à l'aide financière du FNRS et au concours de nombreux collègues, parmi lesquels je me plais tout particulièrement à remercier ici J. Assmann, W. Vivian Davies, Richard A. Fazzini, Peter F. Dorman, Luc et Marc Gabolde, Marsha Hill, Barry J. Kemp, Rolf Krauss, Christian E. Loeben, Stephen Quirke, Kristin Thompson et Dietrich Wildung.

${ }^{5}$ Cf. H. Chevrier, ASAE 26 (1926), p. 121-7 ; ASAE 27 (1927), p. 143-7 ; ASAE 30 (1930), p. 168-9 ; D. C. Forbes, KMT Amarna Letters 3 (1994), p. 46-57.

${ }^{6}$ L'établissement de ce modèle évolutif tripartite de l'art dit amarnien ou atoniste dans sa formulation la plus détaillée et la plus largement admise revient certainement à Cyril Aldred, avec sa contribution sur le sujet dans son célèbre catalogue d'exposition Akhenaton and Nefertiti, 1973.
} 
suggéré d'attribuer à des effigies de Néfertiti'. Il n'est donc pas fortuit, mais résulte assurément d'une intention. Sous prétexte de l'intérêt que l'art atoniste manifeste visiblement pour une représentation moins conceptuelle de la réalité, on a souvent voulu «lire» ces statues sur un mode réaliste, voire hyper-réaliste, d'aucuns cherchant à y reconnaître les signes scrupuleusement et presque cliniquement notés par le sculpteur d'une pathologie particulière dont aurait souffert le futur Akhénaton ${ }^{8}$. De façon assez amusante, c'est l'illustration d'une de ces lectures (hyper-)réalistes des colosses du Gem-pa-Aton qui nous fournit un premier indice patent sur l'un des facteurs explicatifs de ces déformations si impressionnantes qui affectent cette série d'effigies du royal zélateur de l'Aton. En 1930, le médecin G.P.G. Sobhy publia dans le volume 16 du Journal of Egyptian Archaeology un montage photographique (pl. XX) dans lequel il avait remplacé le visage d'une statue d'Amenhotep IV par celui d'un Égyptien contemporain, l'un de ses patients, afin de prouver qu'il est possible qu'un véritable individu soit affublé des traits prêtés au pharaon; son article s'intitule d'ailleurs très explicitement «The Persistence of Ancient Facial Types Amongst Modern Egyptians» ${ }^{9}$. Lorsque 1'on compare attentivement les deux images, celle de la statue et celle du montage photographique, on observe sans difficulté que la position de l'oreille est très différente dans les deux cas. Si, de nos jours, en chirurgie plastique, on oriente conventionnellement la vue de profil d'un visage en alignant horizontalement l'orifice de l'oreille et celui de la pupille ${ }^{10}$, dans l'art pharaonique, le sommet de l'oreille est normalement — et assez naturellement — situé au niveau des sourcils. C'est ce que révèle l'examen des représentations bidimensionnelles, ainsi que celui des statues de taille humaine, ou plus petites. Précisément, si l'on compare le profil des colosses du Gem-pa-Aton avec celui d'une tête du même souverain, mais de taille humaine (Berlin 21.351; ht 26,4 cm), il apparaît que l'oreille des colosses de Karnak est très anormalement haute (pl. XXI). Cette confrontation montre par ailleurs que l'ensemble des traits du visage qui peuvent être reliés par une ligne horizontale sur la tête grandeur nature se retrouvent orientés sur les colosses du Gem-pa-Aton suivant un angle qui augmente graduellement vers le haut, en oscillant entre 14 et 18 degrés. La déformation apparaît donc non seulement intentionnelle, mais aussi systématique, suivant une sorte de règle pré-établie.

7 Soit, essentiellement, le fragment CG 42089, découvert par G. Legrain dans la fouille de la «cour de la cachette»; cf. J. R. Harris, AcOr 38 (1977), p. 9 ; M. Eaton-Krauss, SAK 5 (1977), p. 38, n. 92 ; Ead., CdE 56 (1981), p. 217, n. 3 ; D. Arnold, dans Ead., The Royal Women of Amarna, 1996, p. 17.

${ }^{8}$ Cf., notamment, P. Ghalioungui, ASAE 47 (1947), p. 29-46; C. Aldred et A. T. Sandison, Bulletin of the History of Medicine 36 (1962), p. 293-316 ; J.-Y. Arramon et É. Crubezy, Synoviale 28 (1994), p. 30-3 ; A. Burridge, JSSEA 23 (1996), p. 63-74.

${ }^{9}$ Cf. JEA 16 (1930), p. 3, pl. 3-4. On notera au passage que la démonstration est un peu spécieuse pour ce qui concerne la vue de face (pl. 4 de l'article), puisque le patient du D ${ }^{r}$ Sobhy a été photographié en plongée, ce qui a pour effet d'accentuer l'effet d'allongement de son visage, d'autant que le menton semble, fictivement, se prolonger par la base du cou; lorsque l'on tient compte de ces conditions de prise de vue, la soi-disant ressemblance perd tout son pouvoir de conviction.

10 Je dois cette précision au $\mathrm{D}^{\mathrm{r}}$ B. Lengelé, de l'Université catholique de Louvain-la-Neuve ; cf. D. Laboury, op. cit., p. 648, n. 1927. 
La tête de taille naturelle comparée aux colosses de Karnak ne date pas de la même période du règne d'Amenhotep IV - Akhénaton, puisqu'elle fut découverte dans l'atelier du sculpteur Thoutmose, à Amarna ${ }^{11}$, mais cette différence géographique et chronologique ne peut suffire à rendre compte de la déformation observée. En effet, le complexe atoniste de Karnak comportait assurément des statues de taille humaine ou inférieure, comme l'ont révélé les fouilles de $\mathrm{D}$. Redford et de son équipe à Karnak-est ${ }^{12}$, là où $\mathrm{H}$. Chevrier avait exhumé les sculptures colossales dont il est ici question. Même si l'essentiel de ce matériel archéologique n'est pas encore publié, le magasin dit du Caracol, sur le site de Karnak, conserve une tête fragmentaire (OR 332 ; pl. XXII), évoquée par Marianne Eaton-Krauss ${ }^{13}$, qui date de cette même phase du règne et permet de démontrer la relation qui unit la déformation de la physionomie des colosses du Gem-pa-Aton et leur taille colossale. Cette tête, en grès, aujourd'hui très abîmée, provient d'une statue d'Amenhotep IV - Akhénaton, comme l'indique la présence conjointe de la coiffure khat, de l'urceus et de la titulature du dieu Aton, dans sa première version en cartouches, sur la partie conservée du pilier dorsal. Même si son lieu de découverte précis reste inconnu, sa présence dans un magasin du site de Karnak implique une origine thébaine et, de ce fait, une datation identique à celle des colosses du Gem-pa-Aton, étant donné la brièveté de l'occupation proprement atoniste de Karnak ${ }^{14}$. Les vestiges d'un tenon, ou espace négatif, sur le cou du roi assure qu'il portait la barbe postiche, comme sur les colosses du Gem-pa-Aton. Mais, à l'inverse de ceux-ci, la tête d'Amenhotep IV du Caracol est de taille humaine (ht conservée $39 \mathrm{~cm}$; $\lg$ conservée $33 \mathrm{~cm}$ ). En dépit de la destruction très avancée de son visage, la comparaison de la vue de profil de cette sculpture avec celle du tour de tête de l'atelier de Thoutmose et celle des colosses de Karnak (pl. XXIII) révèle que la structure et les proportions du visage diffèrent avant tout en fonction des dimensions de l'œuvre : en effet, le rendu plastique de l'arrière de la mâchoire accuse un angle nettement plus marqué sur les deux œuvres de

11 Certains indices stylistiques — et en particulier le traitement de ses yeux — permettent en fait de la situer dans la seconde moitié du règne, à partir de l'an 8.

12 Cf. D. B. Redford, dans Hommages à Jean Leclant (BdE 106/1), 1994, p. 487.

13 Cf. M. Eaton-Krauss, SAK 5 (1977), p. 37 (no 29).

14 Rappelons que le premier nom dogmatique de l'Aton n'apparaît en cartouches qu'en l'an 4 (cf. les inscriptions des objets 281a et 291a de la tombe de Toutankhamon suivant la liste de H. Carter ; TAA corpus, p. 131 et 133), que 1'iconographie et le style atonistes ne se mettent en place qu'ensuite (cf. le graffito à droite de celui de Men et Bak à Assouan [L. Habachi, MDAIK 20 (1965), p. 85-92 ; L. O. Congdon, KMT Amarna Letters 2 (1992), p. 42-49] et la stèle de Kia, Royal Scottish Museum acc. n 1956.347 [C. Aldred, JEA 45 (1959), p. 19-22, pl. 3]), mais avant la fin de 1'an 4 (cf. G. Goyon, Nouvelles inscriptions rupestres du Wadi Hammamat, 1957, pl. 25, n 90-1 ; A. Gasse, Égypte, Afrique \& Orient 4 [1994], p. 48-52), que le roi décide de s'installer à Amarna dès la fin du premier tiers de 1'an 5 (W. J. Murnane et Ch. C. Van Siclen III, The Boundary Stelae of Akhenaten [StudEgypt], 1993, 48) et concrétise ce projet en abandonnant son complexe atoniste à Karnak, l'Akhet-en-Aton aux alentours du début de l'an 8 (M. Gabolde, D'Akhénaton à Toutankhamon, 1998, p. 28-30). Je reviendrai prochainement sur l'établissement de cette chronologie dans la biographie d'Akhénaton que je prépare pour la collection Les grands pharaons aux éditions Pygmalion. 
taille humaine et montre que, toute fragmentaire qu'elle soit aujourd'hui, la tête du Caracol présentait assurément une composition générale plus proche de l'étude en plâtre réalisée dans l'atelier du sculpteur Thoutmose à Amarna que de la physionomie déformée des colosses du Gem-pa-Aton, qui lui sont pourtant contemporains et proviennent certainement du même site. La position de l'oreille sur chacune de ces têtes confirme par ailleurs ce regroupement physionomique suivant la taille des statues.

D'autres arguments viennent confirmer cette interprétation des déformations physionomiques des colosses du Gem-pa-Aton comme un effet de gestion volontaire de la perspective. Le premier nous est fourni par le matériel statuaire découvert à Amarna, l'antique Akhet-Aton, où le roi décide de fonder son nouveau lieu de culte de l'Aton dès la fin du premier tiers de l'an 5. Pour ce projet, une quantité impressionnante de statues du souverain sont réalisées afin d'illustrer et d'assurer sa présence permanente dans les temples et palais de la nouvelle cité, statues parmi lesquelles on trouve aussi bien des colosses que des œuvres de taille humaine. Malgré leur état souvent très fragmentaire - qui explique sans doute le faible intérêt qu'elles ont suscité jusqu'il y a peu - et sans entrer dans le détail de leur analyse, on constate aisément que ces sculptures, produites quelques années après leurs homologues de Karnak, corroborent parfaitement la distinction qui vient d'être faite : les visages de taille naturelle présentent des proportions et une morphologie générale identiques à celles de la tête du Caracol et du tour de tête exhumé chez Thoutmose (pl. XXIV) ${ }^{15}$, tandis que les fragments issus de statues colossales affectent exactement les mêmes déformations que les colosses du Gem-pa-Aton de Karnak (pl. XXV) ${ }^{16}$. L'opposition physionomique

\footnotetext{
${ }^{15}$ Cf. les fragments de statues qui ornaient initialement le grand temple d'Aton à Amarna, entassés au sud du mur d'enceinte de ce complexe en une favissa fouillée par H. Carter et W. M. Fl. Petrie en 1891-2 (Petrie, Amarna, p. 18 ; Davies, Amarna III, p. 24). L'essentiel du matériel retrouvé là par les deux archéologues se trouve aujourd'hui au Metropolitan Museum of Art de New York (Hayes, Scepter II, éd.1990, p. 286-287), où Marsha Hill en prépare la publication. Parmi les fragments déjà publiés de cet ensemble qui concernent le sujet envisagé ici, cf. British Museum EA 13366 (don de J. S. Perring, en 1853; ht conservée 15,6 cm ; E. Russmann, Eternal Egypt. Masterworks of Ancient Art from the British Museum, 2001, p. 139-141 [ $\mathrm{n}^{\circ}$ 57]), Metropolitan Museum of Art 26.7.1395 (Edward S. Harkness Gift, 1926; ht conservée 8,2 cm ; Hayes, Scepter II, éd. 1990, p. 286, fig. 174 ; Arnold, op. cit., p. 24 [fig. 16]) ou encore Louvre E 11634 et 11635 (Chr. Barbotin, Les statues égyptiennes du Nouvel Empire. Statues royales et divines, 2007, p. 75, pl. 97). Je tiens à exprimer ici mes plus vifs remerciements à Marsha Hill, qui a si aimablement partagé le fruit de ses recherches sur les fragments du MMA de New York et m'a fourni la vue de face de l'assemblage MMA 1992.227 - 57.180.79 - 57.180.81 (Gift of Sherman and Ruth Lee, 1992 ; Harris Brisbane Dick Fund, 1957), tout en m'autorisant à publier cette tête fragmentaire, qui fera partie de l'étude qu'elle prépare de cet ensemble.

${ }^{16}$ La bouche Petrie Museum UCL 003 (J. Samson, Amarna: City of Akhenaten and Nefertiti, 1972, p. 33-4, pl. 14) s'intègre dans l'ensemble des fragments de statues colossales en quartzite et en granit rose qu'ont mis au jour les fouilles successives de la cour centrale du grand palais d'apparat au centre-ville d'Akhet-Aton. La majorité de ces fragments, à nouveau très abîmés, sont conservés au Musée égyptien du Caire et dans les magasins du SCA sur le site d'Amarna, où j'ai pu les étudier en collaboration avec Kristin Thompson, sur l'aimable invitation de Barry J. Kemp, dans le cadre des activités de l'Amarna Expedition de l'Egypt Exploration Society. Leur analyse révèle l'antique présence, le long des murs bordant cette cour du palais du centre-ville, de colosses en granit rose et en quartzite tout à fait semblables en taille, attitudes et proportions à ceux du Gem-pa-Aton de Karnak. Comme sur ces derniers, la bouche en quartzite du Petrie Museum pré-
} 
observée s'explique donc par la taille des œuvres, et non par leur date de facture ou leur lieu d'origine.

Les talatats remployées à Karnak confortent par ailleurs cette déduction. Ces vestiges du démembrement systématique des édifices construits par Amenhotep IV - Akhénaton pour le culte de l'Aton ont souvent été invoqués pour appuyer l'idée que les déformations qui caractérisent les colosses du Gem-pa-Aton sont parfaitement représentatives du style outrancier et caricatural qui aurait marqué l'émergence de l'ère atoniste à Karnak. Mais, jusqu'aux récentes recherches de Robert Vergnieux sur l'assemblage à grande échelle de ces «pierres du soleil» ${ }^{17}$, on a pratiquement toujours raisonné sur des visages d'Akhénaton ou de Néfertiti qui tiennent sur une et une seule talatat, c'est-à-dire dont la hauteur ne dépasse pas 20 $\mathrm{cm}$. Les regroupements et reconstitutions réalisés par R. Vergnieux permettent aujourd'hui de prendre en considération des visages de plus grandes dimensions et, donc, de facture plus soignée et plus achevée. Apparaissent alors des physionomies dont, d'une part, l'oreille est toujours clairement située à bonne hauteur, au même niveau que le sourcil, et, d'autre part, la déformation est moins accentuée. Je citerai ici le cas très révélateur de la talatat 34-118 du classement de R. Vergnieux, qui provient d'une grande scène figurant l'adoration de l'Aton par le couple royal, qui «embrasse le sol» en présence de la divinité (assemblage A0081; pl. XXVI). Le bloc en question se raccorde avec un autre (34-177) et, ensemble, ils donnent la titulature de la reine : «la grande épouse royale, Néfer-néférou-Aton-Néfertiti, puisse-t-elle vivre infiniment et éternellement». L'intérêt de cette inscription est qu'elle présente la seconde version du nom de la reine, la version allongée de l'épithète intra-cartouche Néfer-néférou-Aton, qui n'apparaît que dans le courant de l'an 6, et que cette dénomination de la reine a été ajoutée a posteriori, soit après la gravure de la scène ${ }^{18}$, qui est donc, de ce fait, antérieure à l'an 6 . Le détail est d'importance, car il permet de dater précisément le relief d'entre l'an 4, année de l'invention du style atoniste et des talatats, et l'an 6, soit une période de temps très courte, qui correspond exactement à celle que l'on peut assigner aux colosses du Gem-pa-Aton. Or, sur ce relief, Néfertiti n'a absolument pas la physionomie déformée de son époux, comme le prédit la théorie du premier style atoniste caricatural de Karnak, mais, au contraire, un visage aux proportions harmonieuses, dont le plus proche parallèle est certainement le fameux buste dit de Berlin, datable de la fin du règne.

Plusieurs conclusions s'imposent donc : $1^{\circ}$ même si l'avènement de l'ère atoniste s'accompagne de l'émergence d'un style résolument neuf par rapport aux traditions égyptiennes,

\footnotetext{
sente un allongement de toute la structure de la mandibule — des lèvres étirées en un sourire irréaliste au menton excessivement fin et accentué - ainsi que des dimensions parfaitement identiques aux éléments correspondants sur les colosses de Karnak. Toute ma gratitude est acquise à Stephen Quirke pour avoir facilité mon étude de cette pièce et m'avoir autorisé à en publier les clichés présentés ici.

${ }_{17} \mathrm{Cf}$. R. Vergnieux, Recherches sur les monuments thébains d'Amenhotep IV à l'aide d'outils informatiques. Méthodes et résultats (CSEG 4), 1999 ; Id. et M. Gondran, Akhénaton ou les pierres du soleil. Akhénaton retrouvé, 1997.

${ }^{18}$ Cf. R. Vergnieux, op. cit., p. 127.
} 
l'opposition contrastée entre style de Karnak ou des premières années de l'Atonisme et style d'Amarna ou de la fin du règne, presque toujours acceptée comme une évidence, est assurément excessive ; $2^{\circ}$ dans les arts bidimensionnels comme dans les arts tridimensionnels, le facteur de la taille de l'œuvre peut induire, dans un sens comme dans l'autre, des déformations importantes, et il faut donc toujours veiller à comparer ce qui peut l'être ; enfin, $3^{\circ}$, les colosses du Gem-pa-Aton de Karnak ne constituent pas l'exemple le plus représentatif du style en vigueur à l'inauguration de l'Atonisme, mais bien une version modifiée de ce style, modifiée en fonction de leur taille et d'un facteur de correction de la parallaxe. Ils doivent donc être observés et analysés en tenant compte de ce facteur de distorsion (ou de correction) et ils posent bel et bien le problème de la prise en considération des déformations de la perspective par l'art égyptien.

La période atoniste étant, d'une certaine manière, la période de toutes les exceptions dans le long fleuve tranquille de l'histoire de la civilisation pharaonique, on pourrait se demander si cette intégration de la parallaxe dans la conception et la réalisation d'une statue colossale est une innovation propre à l'art si singulier de cette époque ou si elle caractérise une plus grande période de l'histoire de l'art égyptien. Le cas des deux statues de l'Akh-menou de Thoutmosis III mentionnées au début du présent article incite bien évidemment à explorer la seconde option ${ }^{19}$. Pour aborder cette question, nous disposons d'un excellent critère, facilement vérifiable et fiable : l'analyse des exemples qui viennent d'être évoqués révèle en effet que sur une vue de profil, la position de l'oreille par rapport au reste du visage est un excellent indicateur de la présence d'une déformation des traits de la statue en fonction de la parallaxe. L'examen de la statuaire pharaonique à l'aune de ce critère permet plusieurs constatations importantes.

Tout d'abord, aucune statue colossale de l'Ancien ou du Moyen Empire ne présente, à ma connaissance, ce type de particularité. Les sculptures de grandes dimensions de ces périodes ne manquent pas et, qu'il s'agisse du grand sphinx de Giza, de la IV ${ }^{\mathrm{e}}$ dynastie, de la tête colossale en granit rose d'Ouserkaf (Le Caire JE 52501 [ht conservée $75 \mathrm{~cm}$ ]), de celles d'Amenemhat III découvertes par E. Naville en 1888 à Boubastis (Le Caire CG 383 [ht conservée $105 \mathrm{~cm}$ ]; British Museum EA 1063 [ht conservée 77,5 cm]) ou des colosses debout de Sésostris I ${ }^{\text {er }}$ à Karnak, Memphis, Tanis et Boubastis, sur toutes ces œuvres, l'oreille conserve la même position que sur les statues de taille humaine, c'est-à-dire que son sommet se trouve au même niveau que les sourcils (pl. XXVII ${ }^{20}$. Le détail est particulièrement

${ }^{19}$ Ce d'autant plus que la majorité des traits caractéristiques de l'art atoniste ont été annoncés par des signes précurseurs pendant le siècle qui précéda l'avènement d'Amenhotep IV - Akhénaton

${ }^{20}$ Pour la statuaire de Sésostris I er évoquée ici, on complètera l'illustration du présent article par L. Gabolde, Le «grand château d'Amon» de Sésostris Ier à Karnak (MAIBL, nouvelle série, 17), 1998, p. 63-70, pl. 20-21, et B. v. Bothmer et J. F. Romano, Musée d'art égyptien ancien de Louxor (BdE 95), 1985, n 25, pour le portique à statues du temple d'Amon à Karnak; et H. Sourouzian, MDAIK 44 (1988), p. 229-254, pl. 62-75, pour les colosses de Boubastis, Tanis et Memphis.

RdE 59 (2008) 
notable et intéressant pour les colosses de Sésostris I ${ }^{e r}$ dont Hourig Sourouzian a magistralement démontré qu'ils avaient été usurpés par Ramsès $\mathrm{II}^{21}$ (pl. XXVIId). En effet, ce dernier en a fait retailler les traits du visage afin de les ajuster à son iconographie, mais, en dépit de ce travail d'adaptation physionomique, ces sculptures se distinguent de toutes les statues colossales réalisées dès l'origine pour représenter Ramsès II (infra, pl. XXX) par la position de leurs oreilles, nettement plus basse, car techniquement impossible à rehausser.

C'est à partir du Nouvel Empire qu'apparaissent donc les premières traces indiscutables de déformation intentionnelle des traits d'une statue colossale en fonction de la perspective. Parmi les plus anciennes sculptures de dimensions colossales de la XVIII dynastie, les piliers osiriaques de Hatchepsout en façade de la troisième terrasse de son temple funéraire à Deir el-Bahari présentent une oreille très légèrement surélevée, dont l'extrémité supérieure atteint la hauteur de la ligne de coiffure, à la base de la couronne sur le front (pl. XXVIIIa). L'altération est assez minimale et pourrait ne pas être vraiment signifiante. Pour le règne de Thoutmosis III, outre l'exemple des deux statues de l'Akh-menou citées en début du présent article, il faut signaler la paire de colosses debout en façade du II ${ }^{\mathrm{e}}$ pylône du grand temple d'Amon-Rê à Karnak, qu'une autre étude de Hourig Sourouzian a permis de ré-attribuer au neveu de Hatchepsout et dont seule la statue méridionale a conservé le visage, bien qu'il soit aujourd'hui très érodé (pl. XXVIIIb) ${ }^{22}$. D'une hauteur de près de 10 mètres, cette œuvre se caractérise, dans la perspective qui nous occupe ici, par une oreille qui dépasse cette fois nettement la ligne de coiffure, ainsi que par une bouche dont le seul élément préservé, la commissure gauche, révèle un sourire plus marqué que celui des sculptures de taille humaine, sourire qui rappelle le profil de la statue de l'Akh-menou CG 594, examinée précédemment (pl. XVIII et XXVIIIc). La déformation très comparable de l'ensemble de la physionomie de ces deux ouvres ${ }^{23}$ interpelle car la première de celles-ci est presque trois fois et demie plus grande que la seconde. Cette ressemblance pose donc le problème de savoir si la correction perspective introduite dans la statuaire colossale pouvait être variable — et si oui, suivant quel(s) critère(s) - ou si elle suivait une sorte de formule toute faite, établie une fois pour toutes, quelle que soit la taille de l'œuvre. Les connaissances mathématiques des anciens Égyptiens, sur lesquels on s'est souvent extasié, dans la foulée des Grecs de l'Antiquité, montre en effet que dans certains cas, la pensée pharaonique pouvait se contenter d'une approximation, jugée pragmatiquement suffisante ${ }^{24}$. La question semble donc pertinente.

La statuaire colossale sous le règne d'Amenhotep III, même si elle est assez abondante, ne permet pas de répondre de manière définitive à cette interrogation car, dans presque tous

\footnotetext{
${ }^{21}$ Soit les colosses de Tanis, Boubastis et Memphis (ibid.).

22 Cf. H. Sourouzian, Karnak 10 (1995), p. 505-529, pl. 1-14 ; D. Laboury, op. cit., p. 319-323.

${ }^{23}$ Même si la taille proportionnelle de l'oreille et l'inclinaison du plan de l'œil varient légèrement.

${ }^{24}$ Cf. la très belle démonstration de Corinna Rossi, Architecture and Mathematics in Ancient Egypt, 2004, notamment p. 60-8 et, de façon générale, toute la partie I de cet ouvrage.
} 
les cas, même si les sculptures peuvent être de taille très variable, l'oreille est légèrement surélevée, presque toujours au niveau du sommet du bandeau qui complète la coiffure sur le front du souverain (pl. XXIX) ${ }^{25}$. L'examen de l'œuvre de l'un de ses émules, Ramsès II, est par contre plus éclairant. En effet, si l'on compare des statues de dimensions différentes, comme les colosses en façade du temple d'Abou Simbel - hauts de plus de 20 mètres la sculpture du roi debout découverte en 1820 sur le site de Mit Rahinah par G.B. Caviglia ou encore la tête de l'un des colosses assis à l'entrée du temple de Louqsor, on constate que l'ampleur de la déformation des traits du visage vers le bas et la hauteur de l'oreille peuvent assurément fluctuer d'un cas à l'autre (pl. XXX). En outre, dans cette confrontation de profils, les deux œuvres qui ont les dimensions les plus proches, soit le colosse de Mit Rahinah et celui dont la tête subsiste en façade du premier pylône du temple de Louqsor, d'une hauteur originale commune d'un peu plus de 15 mètres, présentent les corrections perspectives les plus différentes, ce qui suggère qu'il n'y a pas nécessairement un lien direct entre la taille et l'importance de la déformation.

L'explication de cette variabilité de la déformation perspective des traits des sculptures colossales au Nouvel Empire nous est fournie par l'analyse des statues osiriaques de la première cour du temple mémoriel de Ramsès III à Médinet Habou. À nouveau, la vue de profil de ces sculptures révèle parfaitement, par la position surélevée de l'oreille, une altération de la physionomie en fonction de la perspective ( $\mathrm{pl}$. XXXIb). Cette position anormale de l'oreille est par ailleurs confirmée et bien visible lorsque l'on observe ces statues osiriaques depuis l'extrémité sud de la cour, c'est-à-dire dans les conditions d'éloignement qui réduisent au maximum les effets de parallaxe (pl. XXXIa). Le seul emplacement dans la cour qui permette d'annuler cette déformation, c'est-à-dire d'aligner le sommet de l'oreille avec le niveau des sourcils, correspond, très précisément, à l'axe de la cour, celui que l'on emprunte pour traverser cette dernière et se rendre dans le temple proprement dit (pl. XXXIc). Si l'on se rapproche davantage des statues, la déformation se marque à nouveau, mais dans l'autre sens, c'est-à-dire que l'oreille adopte une position trop basse et, une fois de plus, non naturelle (pl. XXXId). Cet exemple, à lui seul, parce qu'il a conservé son contexte architectural d'origine, montre que la déformation perspective qui affecte les traits d'une statue colossale au Nouvel Empire est fonction, non pas de la hauteur intrinsèque de la sculpture, mais de l'emplacement depuis lequel celle-ci était censée être regardée. Le même type de constatation peut d'ailleurs être fait pour d'autres cas, comme celui des colosses assis en façade du temple d'Abou Simbel, par exemple, dont la déformation des traits révèle qu'ils

\footnotetext{
${ }^{25}$ Même si on note tout de même que le rehaussement de l'oreille est un peu plus important sur la plus grande statue, le colosse méridional de Memnon (haut de près de 16 mètres), que sur les deux autres œuvres illustrées, le buste EA 3 du British Museum (ht conservée $152 \mathrm{~cm}$; ht originale estimée à environ 3,60 mètres, sans la couronne rapportée, d'après H. Sourouzian, Les dossiers d'archéologie 180 [1993], p. 15) et la statue J 131 du Musée de Louqsor (ht conservée 259 cm).
} 
avaient été conçus pour être observés précisément depuis l'extrémité de l'esplanade antique menant au temple (pl. XXXII), ou celui des statues du roi debout entre les colonnes de la grande cour solaire du temple funéraire d'Amenhotep III sur le site actuel du Kôm el-Hettan, dont l'angle formé par la ligne qui rejoint le sommet de l'oreille et le sourcil par rapport à l'horizontale (environ $14^{\circ}$ ) permet de montrer que le point d'observation idéal et théorique de ces statues était une zone d'à peine quelques mètres de profondeur au centre de ladite cour.

À l'analyse, sur une vue de profil, la position de l'oreille au sein du visage d'une statue égyptienne apparaît donc comme un excellent indicateur non seulement de la présence mais aussi de l'importance d'une déformation des traits en fonction de la parallaxe ; elle permet, en outre, de reconstituer, mathématiquement, le contexte de visualisation original de l'œuvre. Ainsi peut-on calculer, par exemple, que, en fonction de l'angle de translation des éléments du visage des statues colossales d'Amenhotep IV érigées dans la cour du Gem-pa-Aton à Karnak — soit approximativement $18^{\circ}$ (supra et pl. XXI) —, ces sculptures avaient été réalisées pour être regardées, en théorie, depuis une distance d'environ 17 coudées, soit un peu moins de 9 mètres $(8,925 \mathrm{~m})$ - alors que, fait notable, elles bordaient une gigantesque cour de 210 mètres (400 coudées) de côté. Dans ces conditions d'observation, le visage, toujours jugé si caricatural, du royal adorateur de l'Aton nous apparaît sous un jour nouveau, suivant l'image qu'en développèrent ses concepteurs et dans une version nettement plus comparable et compatible avec ce que les fragments de statues contemporaines mais de taille humaine permettent de restituer comme portrait officiel du souverain au début de l'ère atoniste (pl. XXXIII) ${ }^{26}$.

Les diverses constatations qui précèdent ont plusieurs implications d'ordre méthodologique et théorique, sur lesquelles je souhaiterais conclure le présent article.

On y a souvent insisté, depuis Platon ${ }^{27}$ jusqu'à nos jours, l'art de l'Égypte antique apparaît comme un véritable archétype des arts qui rejettent et refusent la perspective et ses déformations. Le célèbre ouvrage d'Heinrich Schäfer Von ägyptischer Kunst (1919) est la première étude systématique et théorique d'ampleur qui fut consacrée à ce sujet; l'auteur proposait d'y qualifier de vorgriechisch l'ensemble des formes artistiques qui en cela

${ }^{26}$ La reconstitution proposée ici du visage des statues d'Akhénaton qui ornaient le sanctuaire du grand temple d'Aton à Akhet-Aton - Amarna est réalisée d'après l'assemblage physique des fragments MMA 1992.227 - 57.180.79 - 57.180.81, dû aux recherches de Marsha Hill, que j'ai complété infographiquement par l'ajout de la bouche MMA 26.7.1395, évoquée supra (et dont l'ajustement précis est assuré par la préservation des commissures des lèvres sur les deux œuvres), et de l'œil MMA 66.99.107 (Gallatin collection purchase, Fletcher Fund and the Guide Foundation Gift), dont la provenance amarnienne ne peut être que supposée, mais qui présente exactement la même forme que celui de la tête MMA 1992.227, comme en attestent les traces conservées sur cette dernière. En outre, cet œil a été dédoublé et inversé pour restituer l'œil droit et rendre un visage presque complet à l'œuvre.

27 Sur Platon et l'art égyptien, cf. W. M. Davis, JEA 65 (1979), p. 121-127. 
s'apparentent au système de représentation pharaonique. Dans la tradition occidentale, les Grecs de l'époque dite classique passent effectivement pour les inventeurs de la figuration perspective et, pour ce qui concerne la statuaire, la littérature de cette période se fait parfois l'écho d'une réflexion théorique sur l'altération des traits que subit une sculpture colossale sous l'effet de la parallaxe ${ }^{28}$. Mais comme me l'a fait remarquer l'historien de la sculpture grecque Andrew Stewart ${ }^{29}$, la disparition de tous les colosses d'époque classique rend pratiquement invérifiable l'impact pragmatique de ces considérations d'ordre théorique sur l'art statuaire en Grèce aux $\mathrm{V}^{\mathrm{e}}$ et $\mathrm{IV}^{\mathrm{e}}$ siècles avant notre ère. L'historien de l'art égyptien semble se trouver quant à lui dans une situation inverse : aucune trace écrite d'une réflexion théorique sur ce problème n'a subsisté dans ce qui nous est parvenu de la littérature pharaonique ${ }^{30}$, même si les derniers exemples que nous venons d'examiner impliquent une approche mathématique, donc théorique ${ }^{31}$, de la parallaxe et de ses effets ; par contre, les œuvres sont conservées et elles démontrent l'existence d'une prise en considération effective et visible de la perspective dans la statuaire égyptienne de grandes dimensions, dès le Nouvel Empire.

Il convient sans doute de s'interroger sur la manière dont les sculpteurs égyptiens ont pu réaliser de telles déformations, aussi efficaces, c'est-à-dire qui corrigent effectivement les altérations dues à la parallaxe en fonction d'un point de visée de toute évidence déterminé à l'avance. En effet, la précision de la correction des traits des colosses de Médinet Habou, d'Abou Simbel, du Kôm el-Hettan ou du Gem-pa-Aton de Karnak — précision qui est telle que l'on peut en reconstituer, en sens inverse, les conditions architecturales de visualisation des œuvres - et les dimensions mêmes de ces statues rendent impossible toute hypothèse d'une approche purement et exclusivement empirique du problème par les sculpteurs égyptiens. Par ailleurs, il est aujourd'hui parfaitement établi que les mathématiques pharaoniques ignoraient le concept d'angle, qui nous a servi à quantifier les altérations observées. Pour mesurer une inclinaison, les anciens Égyptiens utilisaient un autre principe, celui du sqd. Ce dernier peut être défini comme le déplacement horizontal d'un

28 Cf. A. Stewart, Greek Sculpture. An Exploration, 1990, p. 20 (Platon, Le sophiste, 235E).

${ }^{29}$ Dans un e-mail qu'il m'a adressé le 4 décembre 2000. Ma plus profonde gratitude est acquise à Andrew Stewart pour l'intérêt qu'il a porté à mes recherches en cette matière et pour l'amabilité avec laquelle il a bien voulu répondre à mes interrogations.

${ }^{30}$ Cette absence ne doit en réalité pas surprendre puisque les anciens Égyptiens ne nous ont laissé aucun traité théorique à propos de leur art (à ce sujet, cf. J. Baines, Cambridge Archaeological Journal 4 [1991], p. 74-5), principalement en raison de la limitation de leur littérature, de ce qui, selon eux, était destiné à durer sous une forme écrite, à quelques genres seulement, parmi lesquels un tel discours sur l'art n'aurait pu trouver la moindre place. Il y a néanmoins quelques traces indirectes de l'existence d'une approche théorique ou théorisante de l'art ; cf. par exemple, en matière de style, le dialogue analysé par J. Assmann, in I. Gamer-Wallert, W. Helck (éd.), Gegengabe. Festschrift für Emma Brunner-Traut, 1992, p. 43-60.

31 «Mathématique» et «théorique» étant pris ici dans le sens que l'on peut donner à ces mots lorsqu'on les utilise à propos de la pensée pharaonique ; à ce sujet, cf. C. Rossi, op. cit., passim. 
plan incliné par rapport à une ligne verticale d'une coudée de haut, tirée à partir de ce plan $^{32}$. Un tel procédé de caractérisation mathématique d'une inclinaison ou d'un angle est très facile à mettre en œuvre pour des applications architecturales et il fut manifestement fort utilisé dans ce contexte en Égypte antique. Si l'on veut transposer la méthode de mesure du sqd au problème qui nous occupe, la ligne de référence doit être non plus verticale, mais horizontale. En effet, les cas de Médinet Habou, d'Abou Simbel et du Kôm elHettan analysés précédemment montrent que le plan géométrique d'alignement pris en compte était celui qui relie le sommet des oreilles aux sourcils, plan qui est horizontal sur les statues de taille humaine et sur les représentations bidimensionnelles et par rapport auquel il y a déformation. Si l'on mesure donc les déformations observées ci-dessus suivant ce concept pharaonique, on constate qu'elles correspondent toutes exactement à des fractions dont le numérateur est l'unité, 1/2,1/3,1/4, 1/6 et 1/8 (voir l'annexe en fin de cet article), soit des fractions qui sont parmi les rares que les mathématiques égyptiennes toléraient et qui se retrouvent très fréquemment dans les rapports de proportions des compositions artistiques de l'Égypte antique ${ }^{33}$. La coïncidence ne semble pas pouvoir être fortuite. Et, manifestement, les concepts mathématiques dont se servaient les anciens Égyptiens permettaient de calculer à l'avance les corrections perspectives que nous avons constatées ${ }^{34}$.

En tout cas, quelles qu'aient été la ou les techniques réellement utilisées, et au-delà du fait que les Grecs semblent une fois de plus avoir été devancés de près d'un millénaire en cette matière par les anciens Égyptiens, auxquels ils prétendaient eux-mêmes tant devoir, le phénomène observé est particulièrement intéressant car il démontre que les artistes pharaoniques avaient, au moins à partir du Nouvel Empire, une réelle connaissance de la perspective, de ses modalités d'application et de ses effets, ce qui implique, en corollaire, que le rejet de la perspective qui caractérise effectivement l'art égyptien résulte, sans nul doute possible — à tout le moins à partir du Nouvel Empire — d'un choix conscient et volontaire et qu'il est donc signifiant, comme l'avait parfaitement compris H. Schäfer, il y a déjà presque un siècle.

32 Sur la notion de sqd (Wb IV, 309, 20) et son utilisation concrète, cf. Ead., op. cit., p. 185-186, 192-196, 203-204, 208, 213 et 236; et D. Arnold, Building in Egypt. Pharaonic Stone Masonry, 1991, p. 11-14. Dans notre système de concepts mathématiques, ce principe trouve en fait son équivalent dans la notion de pente en topographie (soit la différence entre la hauteur maximale et la hauteur minimale d'une pente divisée par la distance horizontale entre ces deux niveaux et exprimée en pourcentages), à la différence que la référence n'est pas l'horizontale, comme pour la pente, mais la verticale ; comme nous allons le voir, il n'est pas à exclure que le principe du sqd pouvait être transposé pour fonctionner exactement comme celui de notre pente topographique. C'est un plaisir pour moi que de remercier ici Corinna Rossi pour l'amabilité avec laquelle elle m'a apporté son concours et ses suggestions dans l'analyse de ces questions.

33 Cf., par exemple, J.-Fr. Carlotti, Karnak 10 (1995), p. 65-94, et C. Rossi, op. cit., passim.

${ }^{34}$ Comme en architecture (Ead., op. cit., p. 96-147, 174-6), il ne faut pas exclure que cette planification théorique et mathématique de l'œuvre était ensuite complétée et validée par une maquette préalable à la véritable réalisation de la statue. Dans cette perspective, la petite tête d'Amenhotep IV - Akhénaton MMA 21.9.17 (Gift of Edward S. Harkness, 1921; grès; en provenance d'Amarna; ht conservée 9,2 cm), que W. C. Hayes identifie comme une étude de sculpteur (Scepter II, éd. 1990, p. 282) et qui présente une physionomique très proche de celle des colosses du Gem-pa-Aton de Karnak, pourrait, peut-être, en être un indice. 
Enfin, le fait que cette intégration des problèmes de parallaxe dans la conception et la réalisation des statues colossales se manifeste au Nouvel Empire n'est, bien entendu, pas non plus un effet du hasard. Nous l'avons vu, replacé dans son contexte monumental, le phénomène correspond en réalité à une prise en considération du spectateur de l'œuvre, ce qui, en soi, constitue une innovation importante par rapport à l'esprit originel de l'art égyptien, puisque celui-ci contient, en théorie, son propre spectateur, celui auquel il est destiné, et ignore souverainement celui qui lui est externe ${ }^{35}$. Le développement de cette interface visuelle avec le spectateur-visiteur et l'introduction de plus en plus importante du visuel et de la visualité dans le système de l'image caractérisent précisément l'évolution artistique au Nouvel Empire. Apparaissent ainsi des effets de perspective, voire de véritable trompel'œil, dans l'architecture ${ }^{36}$, tandis que les arts bidimensionnels, et en particulier la peinture, exploitent de plus en plus leurs capacités de communication visuelle avec le spectateur ${ }^{37}$. Ces changements, qui affectent en réalité des éléments fondamentaux et fondateurs de l'art égyptien, s'inscrivent et prennent leur véritable sens dans une mutation plus vaste de la pensée pharaonique, qui, avec le Nouvel Empire, va accorder une place plus grande à la perception, au visuel et au visible, et à l'individualité, ouvrant notamment la voie à une approche plus phénoménologique et plus rationaliste de la réalité, nouvelle approche qui explosera littéralement dans l'épisode atoniste ${ }^{38}$. Mais cette relation entre système de représentation plastique et système de représentation métaphysique constitue en soi l'objet d'une autre étude, trop vaste pour ces quelques pages et sur laquelle je me promets de revenir prochainement.

\footnotetext{
35 À ce propos, cf. R. Tefnin, Annales d'Histoire de l'Art et Archéologie 5 (1983), p. 10-12 (article republié dans GM 79 [1984], p. 55-72). Pour le cas précis analysé dans les pages qui précèdent, c'est-à-dire celui de la statuaire colossale, on ne manquera pas, par ailleurs, de mettre en relation cette communication qui s'installe visuellement entre la statue et son spectateur avec le développement, au Nouvel Empire, du culte rendu aux effigies colossales de pharaon ; à ce propos, cf., entre autres références, R. el-Sayed, BIFAO 79 (1979), p. 155-166 ; S. Bickel, BIFAO 102 (2002), p. 63-90.

${ }^{36}$ Cf. l'étude de base sur ce sujet, due à A. Badawy, in Studies in Honor of John A. Wilson (SAOC 35), 1969, p. 15-23. On peut, par ailleurs, citer quelques exemples peut-être plus probants, comme celui de l'architecture de la salle large de la tombe thébaine 29 (du vizir Amenemopé, sous Amenhotep II), dont l'étude qu'y mène la Mission Archéologique dans la Nécropole Thébaine de l'Université Libre de Bruxelles a montré que l'écartement des (10) piliers s'accentue au fur et à mesure que l'on s'éloigne de l'axe de la tombe, afin d'alléger l'effet d'écrasement provoqué par la parallaxe, les piliers paraissant alors équidistants au spectateur qui traverse la salle en suivant cet axe pour se rendre dans le fond de la tombe.

${ }^{37}$ Sur un plan stylistique, on songera à tous les effets de texture qui se font jour dans la décoration picturale - mais

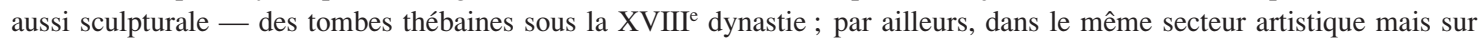
un plan iconographique, il y a manifestement une très forte volonté de communiquer visuellement avec le visiteur, afin de faire passer un message particulier sur l'identité du défunt ; à ce sujet, on se reportera à l'étude de M. Hartwig, Tomb Painting and Identity in Ancient Thebes, 1419-1372 BCE (MonAeg 10), 2004.

38 Ainsi que l'ont bien montré les travaux de Jan Assmann sur l'idéologie atoniste et son contexte intellectuel et philosophique, notamment dans Re und Amun. Die Krise des polytheistischen Weltbilds im Ägypten der 18.-20. Dynastie (OBO 51), 1983, ou Akhanyati's Theology of Light and Time (Proceedings. The Israel Academy of Sciences and Humanities VII/4 [= p. 143-176]).
} 
Annexe: calcul des angles de déformation des traits de statues colossales du Nouvel Empire en sqd.

Les mesures suivantes ont été réalisées à partir de vues de profil des statues concernées, sur lesquelles une ligne a été tracée pour relier le sommet de l'oreille au sourcil. L'inclinaison de la pente ainsi matérialisée a été quantifiée suivant le concept égyptien du sqd (cf. supra), mais en prenant une référence horizontale, c'est-à-dire en mesurant le déplacement vertical de ladite pente par rapport à une unité en longueur horizontale ${ }^{39}$. On obtient ainsi un $s q d$ que je propose d'appeler $s q d$ vertical, par opposition au sqd habituellement utilisé en architecture égyptienne, qui sert à déterminer le déplacement horizontal d'un mur présentant un fruit. Il convient cependant de noter que le $s q d$ dit vertical n'est rien d'autre que l'inversion du sqd architectural ou horizontal, puisqu'il correspond à une fraction dont le dénominateur serait ce dernier et le numérateur 1 ; en d'autres termes, un sqd vertical d' $1 / 4$ équivaut à un $s q d$ horizontal de $4,1 / 8-8$, etc. Pour calculer ce même angle dans notre système mathématique, soit en degrés, il suffit de suivre la formule suivante : $\tan \alpha=\mathrm{ht} / \mathrm{lg}$ (soit la valeur du sqd dit vertical).

\begin{tabular}{|l|c|}
\hline Statues colossales & $\begin{array}{c}\text { Sqd } \\
\text { vertical }\end{array}$ \\
\hline Colosses osiriaques C du temple de Hatchepsout à Deir el-Bahari & $1 / 8$ \\
\hline Le Caire CG 594 et Karnak MPA.T3.st1 (Thoutmosis III) & $1 / 4^{40}$ \\
\hline Karnak, colosse devant le môle sud du II ${ }^{\mathrm{e}}$ pylône (Thoutmosis III) & $1 / 4^{41}$ \\
\hline Louqsor J 131 (Amenhotep III) & $1 / 3^{42}$ \\
\hline British Museum EA 3 (Amenhotep III) & $1 / 4^{43}$ \\
\hline Colosses debout de la cour solaire du Kôm el-Hettan (Amenhotep III) & $1 / 4$ \\
\hline Colosses du Gem-pa-Aton (Amenhotep IV) & $1 / 3$ \\
\hline Berlin 1479 (Ay) & $1 / 3$ \\
\hline Le Caire JE 59869 (Ay) & $1 / 3$ \\
\hline
\end{tabular}

39 Cette mesure est très facile à effectuer sur des images digitales avec le logiciel d'Adobe Photoshop : il suffit de tracer avec l'outil «rectangle de sélection» un rectangle dont les angles opposés se situent sur la pente à mesurer et dont le côté horizontal correspond à une unité (quelle qu'elle soit, d'ailleurs); la longueur de l'autre côté est alors immédiatement déterminée par le logiciel dans la fenêtre nommée «infos».

40 À partir de ce $s q d$, en tenant compte de la hauteur originale des deux statues $(280 \mathrm{~cm})$ et en estimant le niveau de l'œil d'un spectateur moyen de l'époque à environ $175 \mathrm{~cm}$ au-dessus du sol, on peut calculer que la distance d'observation théorique de ces statues était prévue à 8 coudées, soit $421 \mathrm{~cm}$ de distance, c'est-à-dire contre le mur opposé de la salle où se dressaient les deux sculptures, salle dont la profondeur était de 9 coudées (J.-Fr. Carlotti, L'Akh-menou de Thoutmosis III à Karnak. Étude architecturale, 2001, p. 113-114, 199-200, pl. 2 et 33).

${ }^{41}$ Suivant le calcul évoqué à la note précédente, on peut estimer la distance théorique de visualisation de ce colosse et de son jumeau, sans doute originellement situés en façade de la cour des fêtes à l'entrée du temple d'Amon-Rê de Karnak sous Thoutmosis III, à 52 coudées, soit approximativement 27 mètres.

${ }^{42}$ Estimation de la distance théorique de visualisation en fonction de la taille reconstituée de l'œuvre $( \pm 295 \mathrm{~cm})$ : 7 coudées, soit 3,675 mètres.

${ }^{43}$ Estimation de la distance théorique de visualisation en fonction de la taille reconstituée de l'œuvre, sans sa couronne $( \pm 360 \mathrm{~cm}[$ supra, n. 25]) : 14 coudées, soit 7,35 mètres. 


\begin{tabular}{|l|l|}
\hline Statues assises en façade du temple de Louqsor (Ramsès II) & $1 / 6$ \\
\hline Colosse debout de Mit Rahinah (Ramsès II) & $1 / 2^{44}$ \\
\hline Statues assises en façade du temple d'Abou Simbel (Ramsès II) & $1 / 3$ \\
\hline $\begin{array}{l}\text { Tête colossale en diorite dans la 2 } \\
\text { Amenhotep III?) }\end{array}$ & $1 / 4$ \\
\hline Colosse dit d'Osymandias au Ramesséum (Ramsès II - Amenhotep III?) & $1 / 2^{45}$ \\
\hline Colosses osiriaques de la 1 ${ }^{\text {re }}$ cour du temple de Médinet Habou (Ramsès III) & $1 / 3$ \\
\hline
\end{tabular}

\section{Addendum}

Alors que le présent article était pratiquement sous presse, Christophe Thiers a eu l'amabilité d'attirer mon attention sur une stèle monumentale $(617 \mathrm{~cm}$ de haut) d'époque ptolémaïque, dont l'étude a révélé qu'elle présente, elle aussi, des déformations notables, tant dans sa forme générale que dans celle des lignes de son inscription, afin de réduire les effets de la parallaxe pour un spectateur positionné sur le sol, près de sa base : cf. la contribution de L. Tamborero dans Chr. Thiers, La stèle de Ptolémée VIII Évergète II à Hérakléion (Alexandrie Musée Maritime Csa 529) (Oxford Center for Maritime Archaeology: Monograph 4), 2008, p. XVIII-XIX. Je tiens à remercier vivement Chr. Thiers de m'avoir fait part de ces informations encore inédites.

\section{Résumé / Abstract}

Les déformations qui affectent la physionomie des statues colossales du Nouvel Empire suggèrent que l'art pharaonique pourrait avoir pris en considération les effets de la parallaxe et les distorsions dues à la perspective. Cette question et ses implications méthodologiques et théoriques sont examinées, avec une attention particulière prêtée aux colosses d'Amenhotep IV découverts à Karnak.

The deformation which affects the physiognomy of colossal sculptures of the New Kingdom suggests that Ancient Egyptian Art could have taken into account the parallax effects or distortions due to perspective. This question and its methodological and theoretical implications are investigated, with a special focus on the colossi of Amenhotep IV from Karnak.

\footnotetext{
44 Estimation de la distance théorique de visualisation en fonction de la taille reconstituée de l'œuvre, sans sa couronne ( $\pm 15,75 \mathrm{~m}-30$ coudées) : 53 coudées, soit environ 28 mètres.

45 Cf. n. 44.
} 


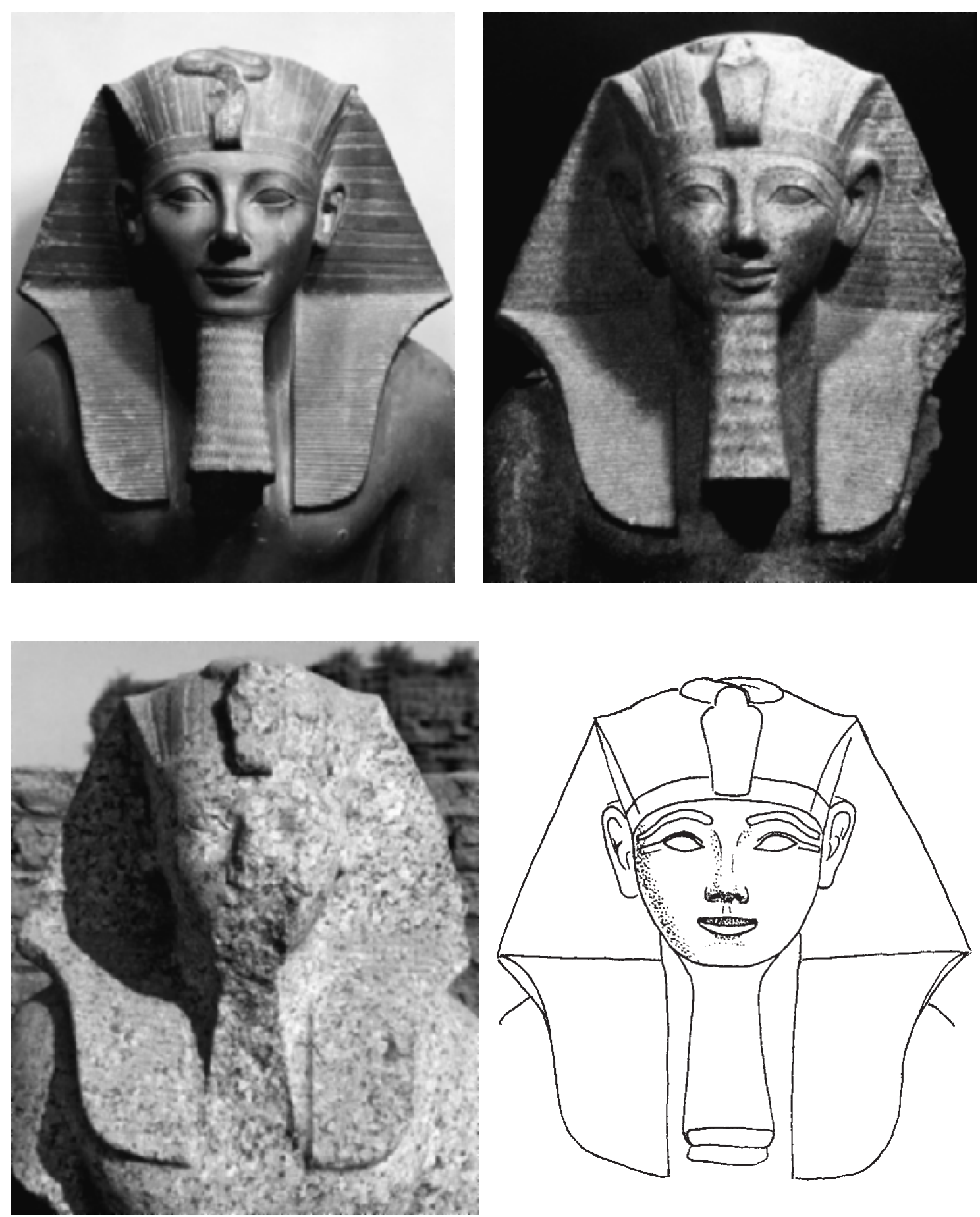

Vue de face, à hauteur des yeux, des statues Louqsor J2, Le Caire CG 594 et Karnak MPA.T3.st1 (et reconstitution graphique), d'après D. Laboury, La statuaire de Thoutmosis III (AegLeod 5), p. 161, 174-175.

D. Laboury, Colosses et perspective. De la prise en considération de la statuaire pharaonique de grandes dimensions au Nouvel Empire. 


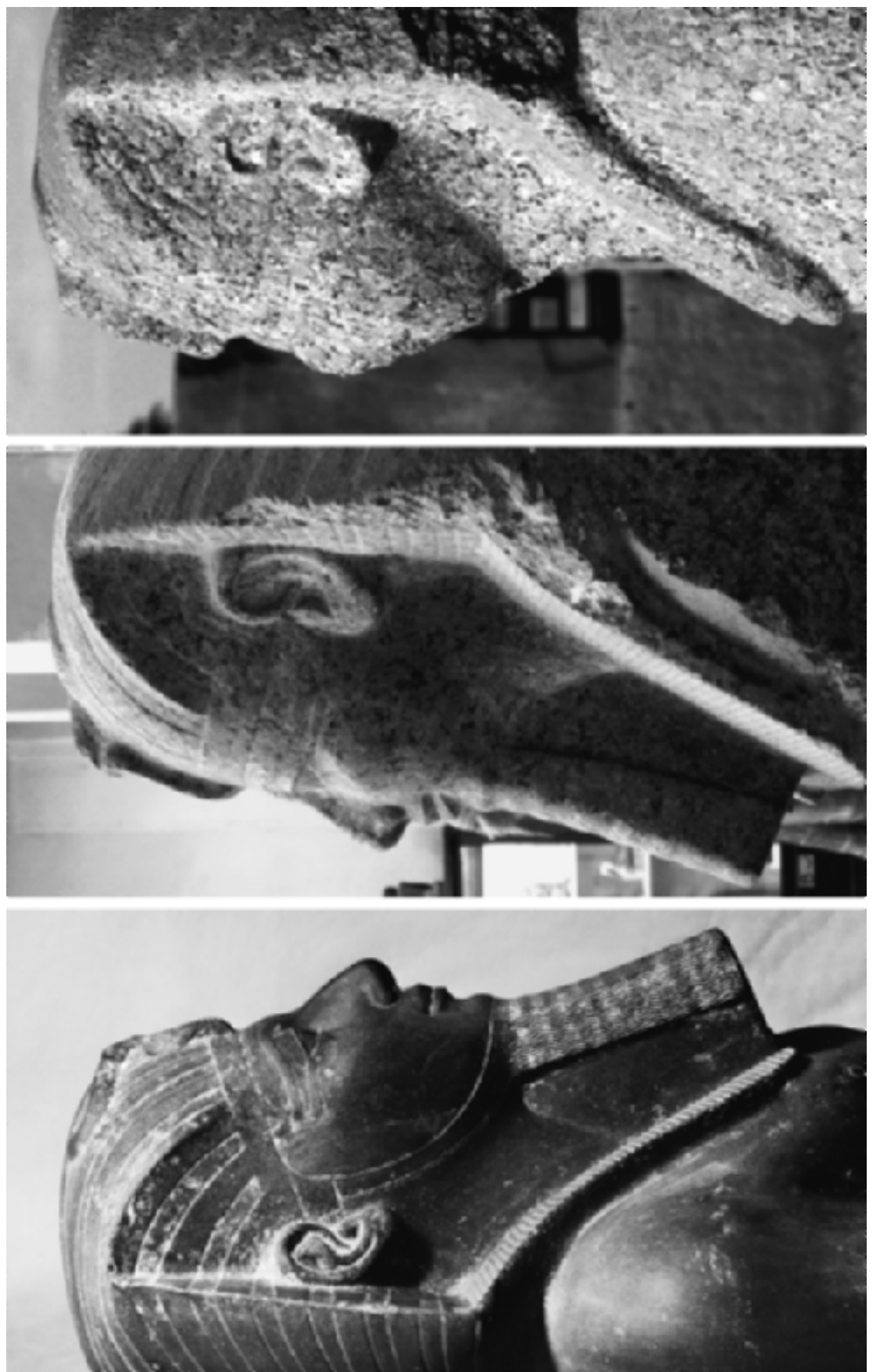

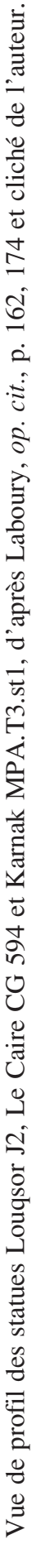

D. Laboury, Colosses et perspective. De la prise en considération de la statuaire pharaonique de grandes dimensions au Nouvel Empire. 


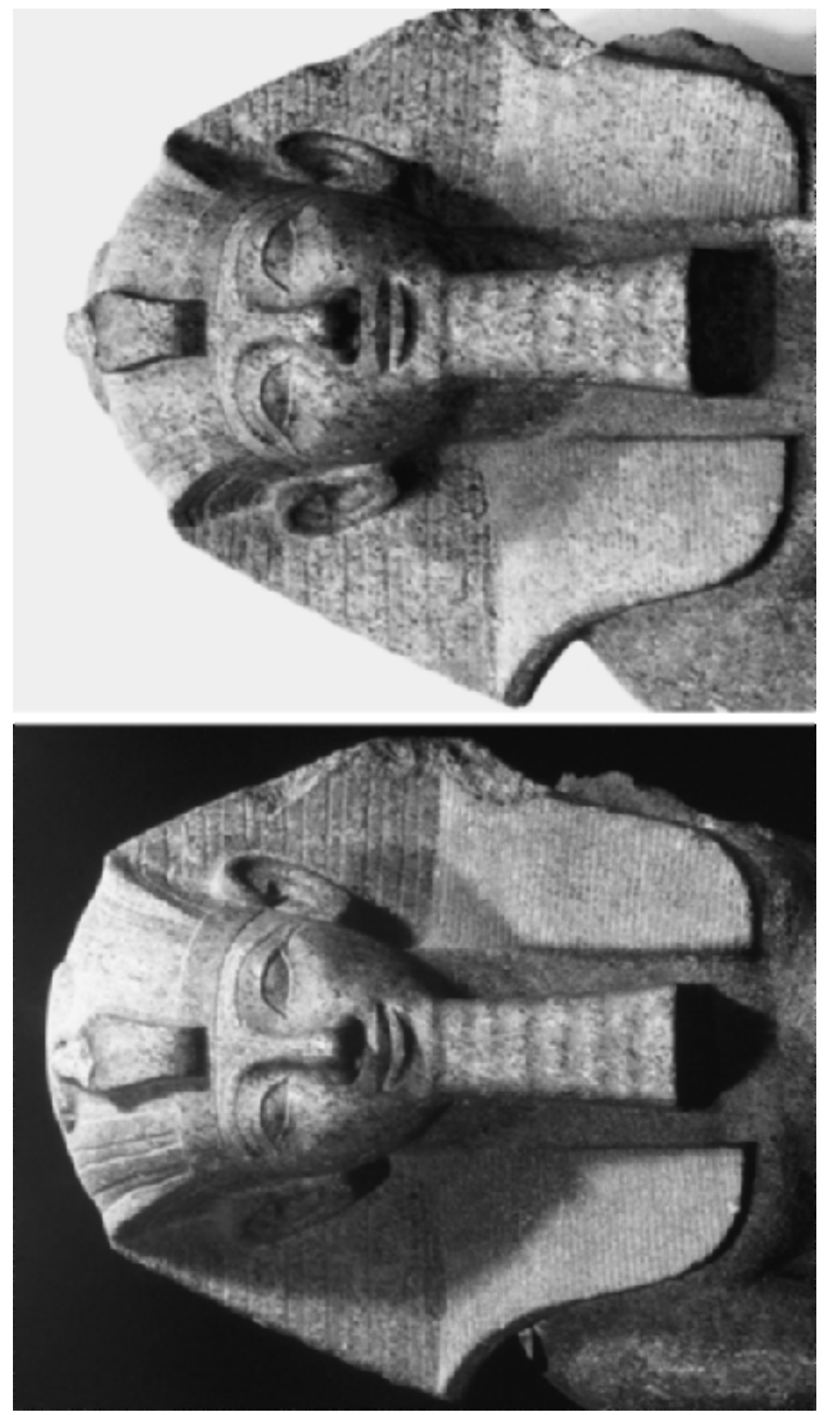

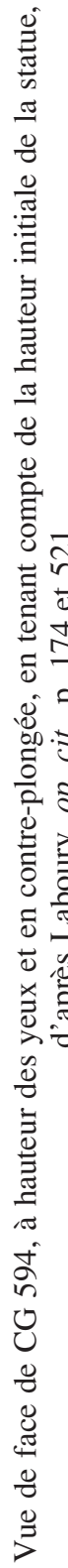

D. Laboury, Colosses et perspective. De la prise en considération de la statuaire pharaonique de grandes dimensions au Nouvel Empire. 



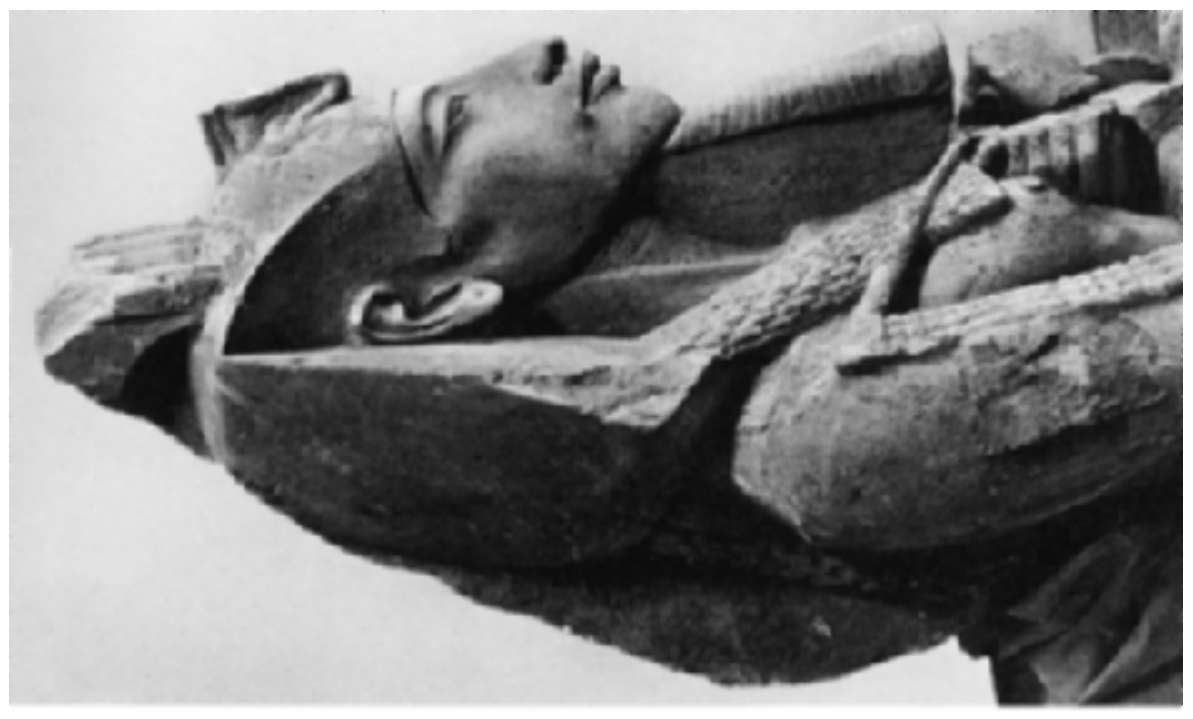

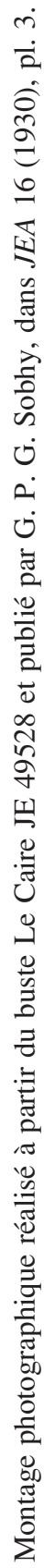

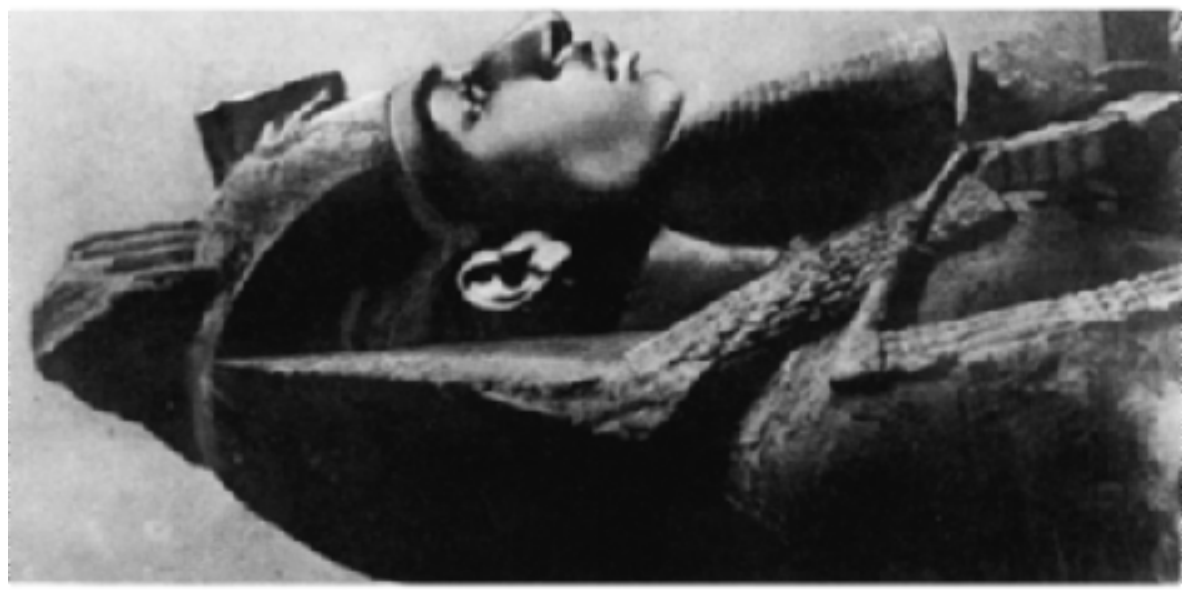

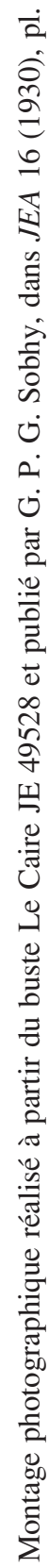

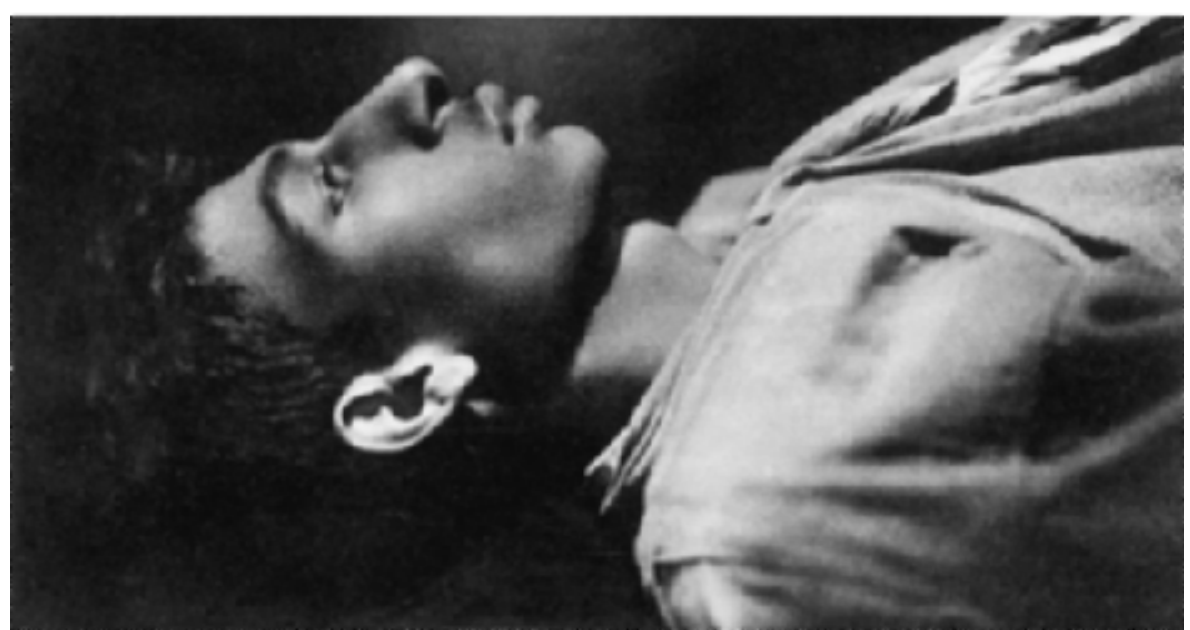

D. Laboury, Colosses et perspective. De la prise en considération de la statuaire pharaonique de grandes dimensions au Nouvel Empire. 


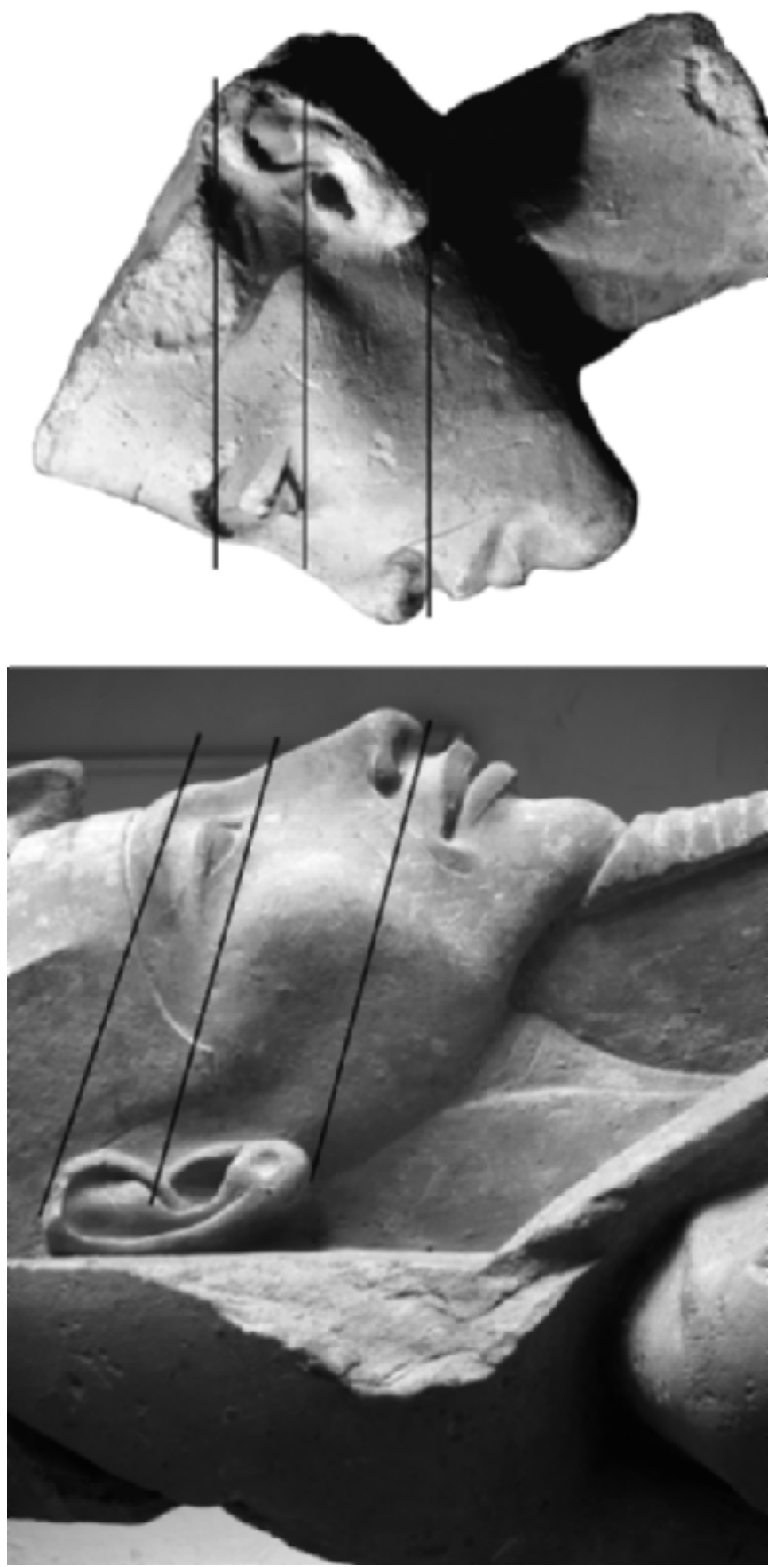

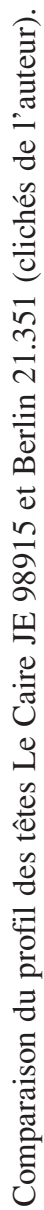

D. Laboury, Colosses et perspective. De la prise en considération de la statuaire pharaonique de grandes dimensions au Nouvel Empire. 


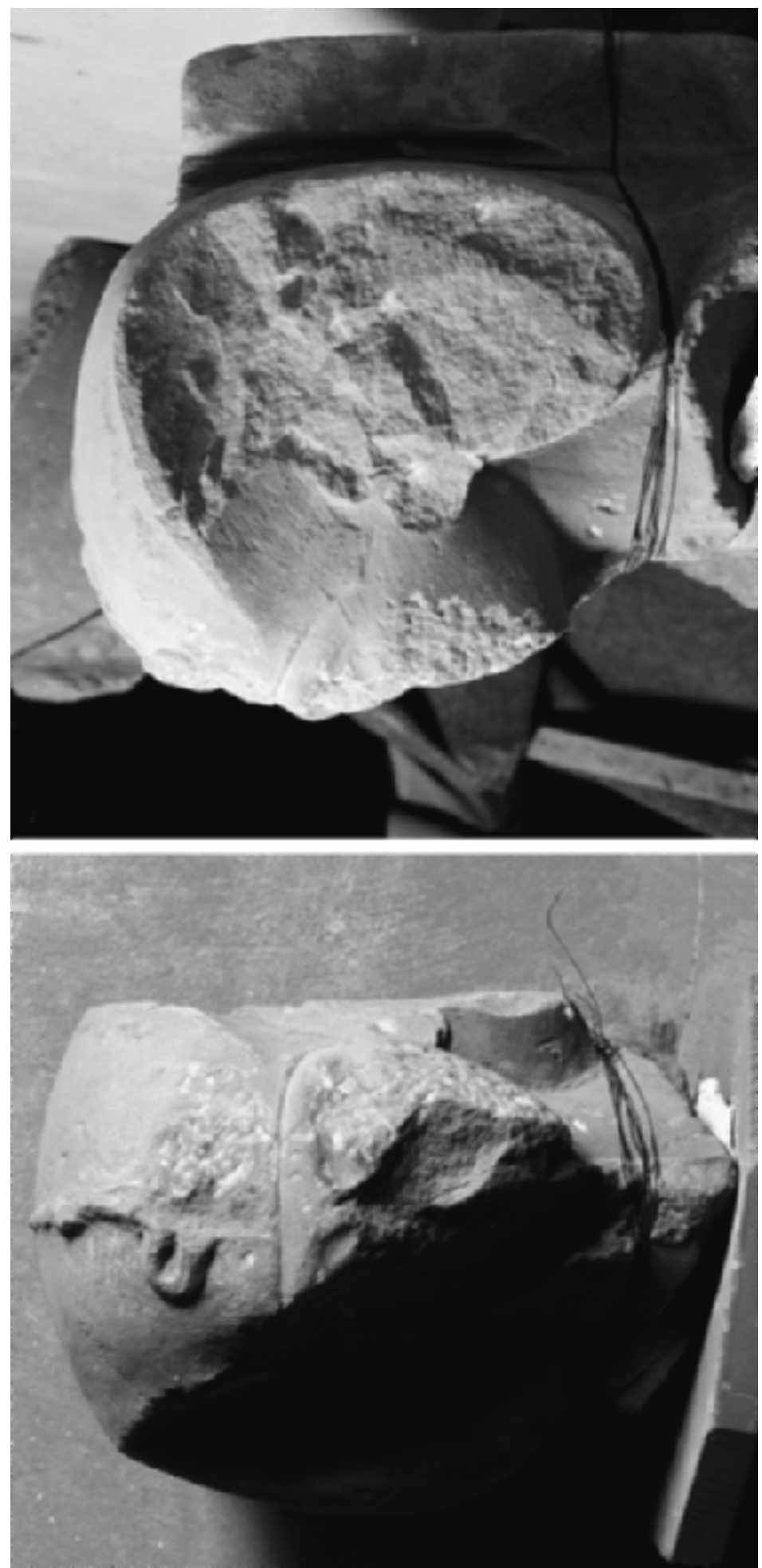

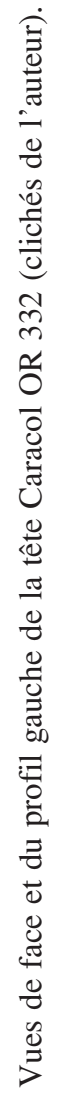

D. Laboury, Colosses et perspective. De la prise en considération de la statuaire pharaonique de grandes dimensions au Nouvel Empire. 



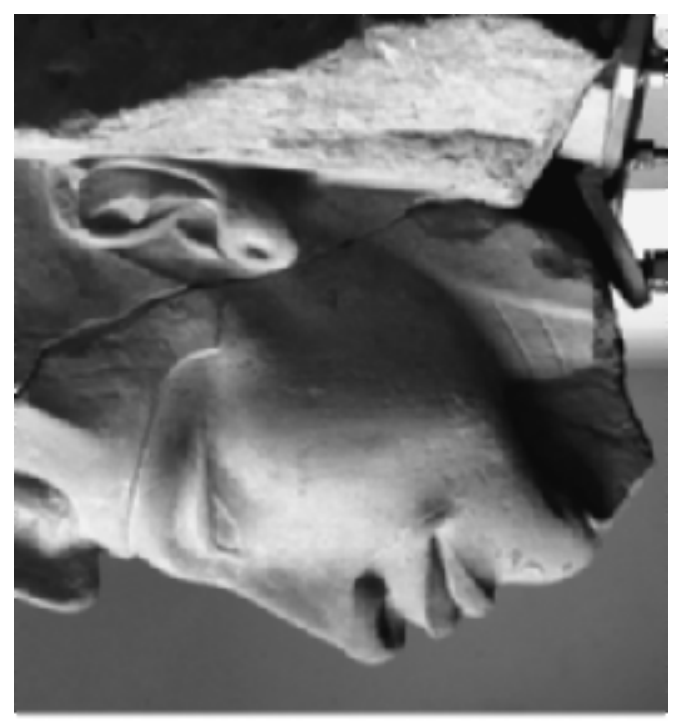

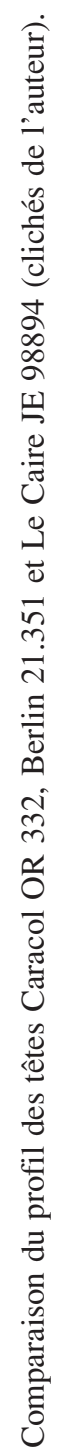

D. Laboury, Colosses et perspective. De la prise en considération de la statuaire pharaonique de grandes dimensions au Nouvel Empire. 



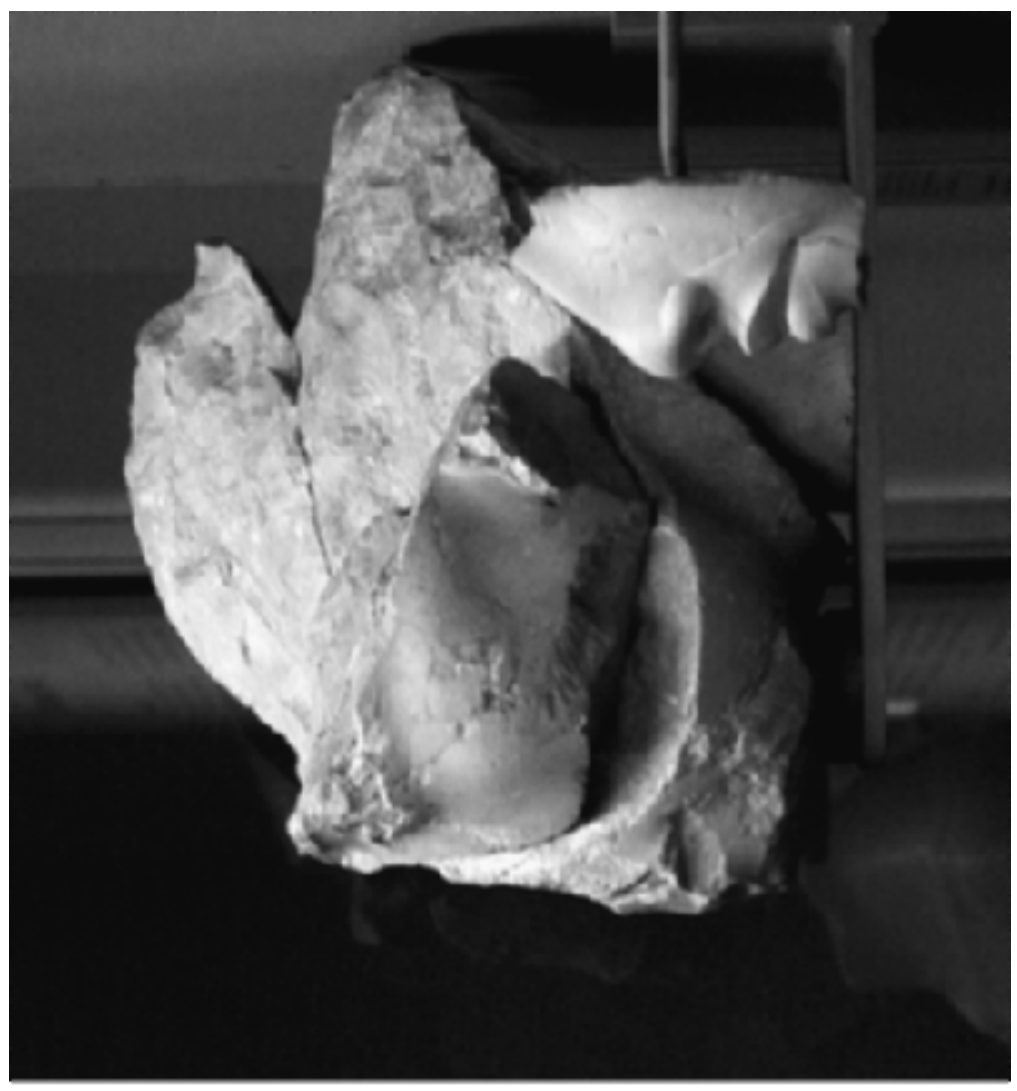

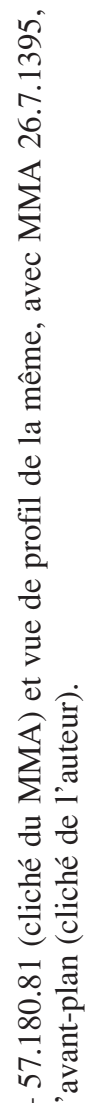

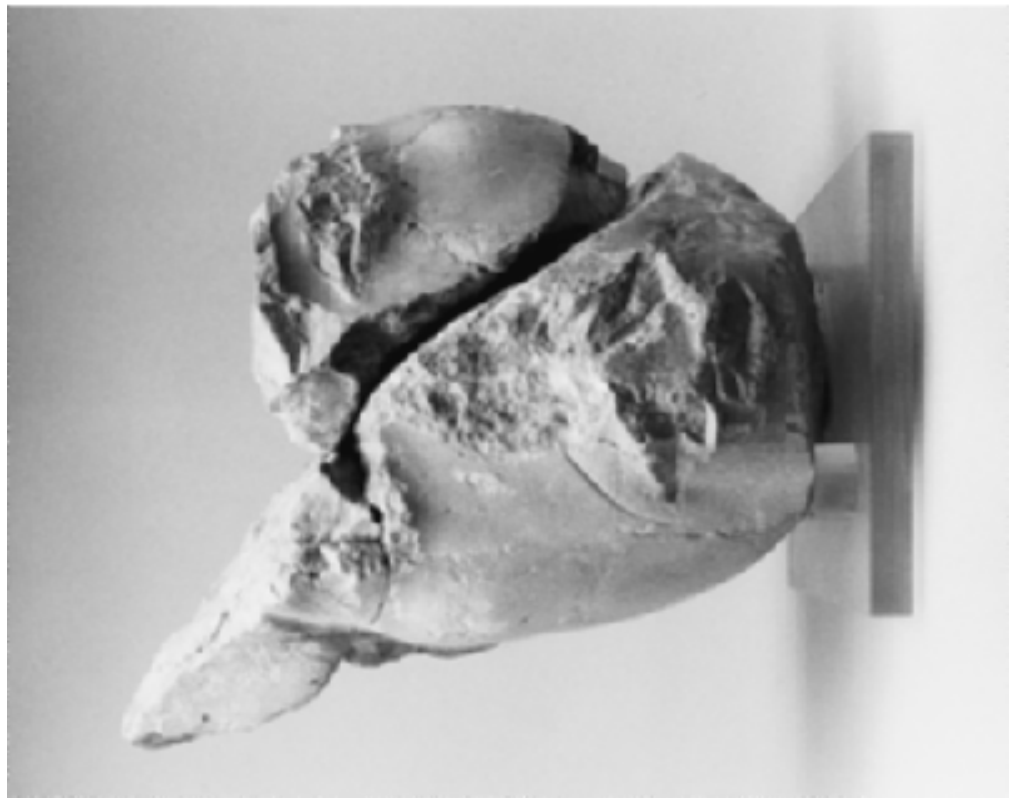

$2 \pi$

$\infty$

in

ลิ

ริ

วิ

$\sum_{\Sigma}^{\mathbb{2}}$

过

$\pi$

$\frac{8}{8}$

巡

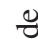

$\stackrel{0}{5}$

D. Laboury, Colosses et perspective. De la prise en considération de la statuaire pharaonique de grandes dimensions au Nouvel Empire. 



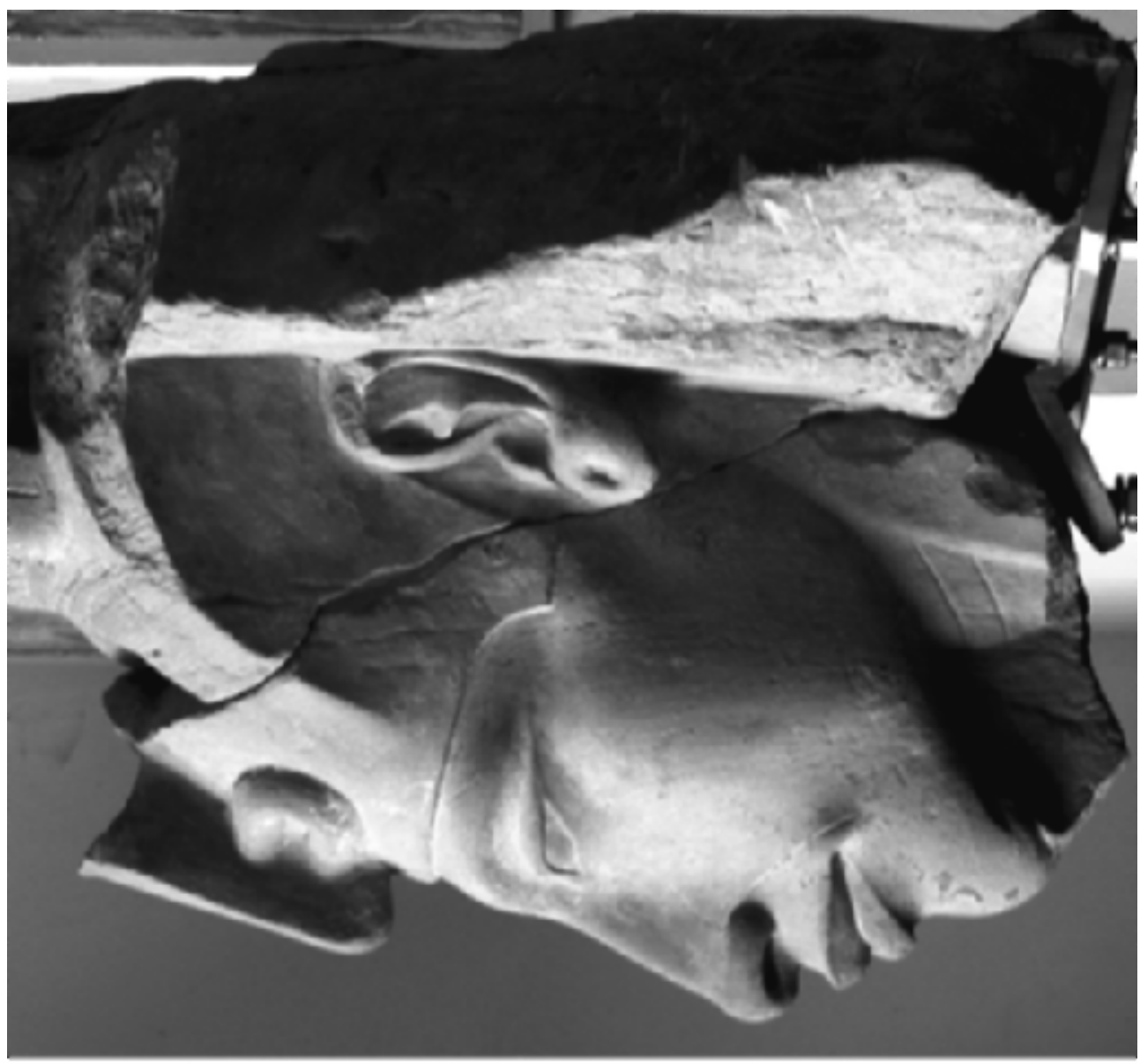

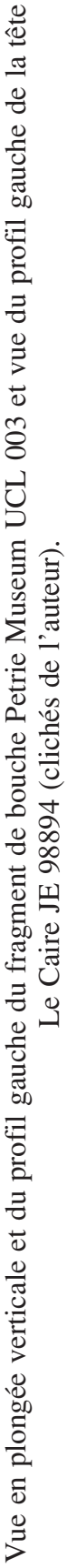
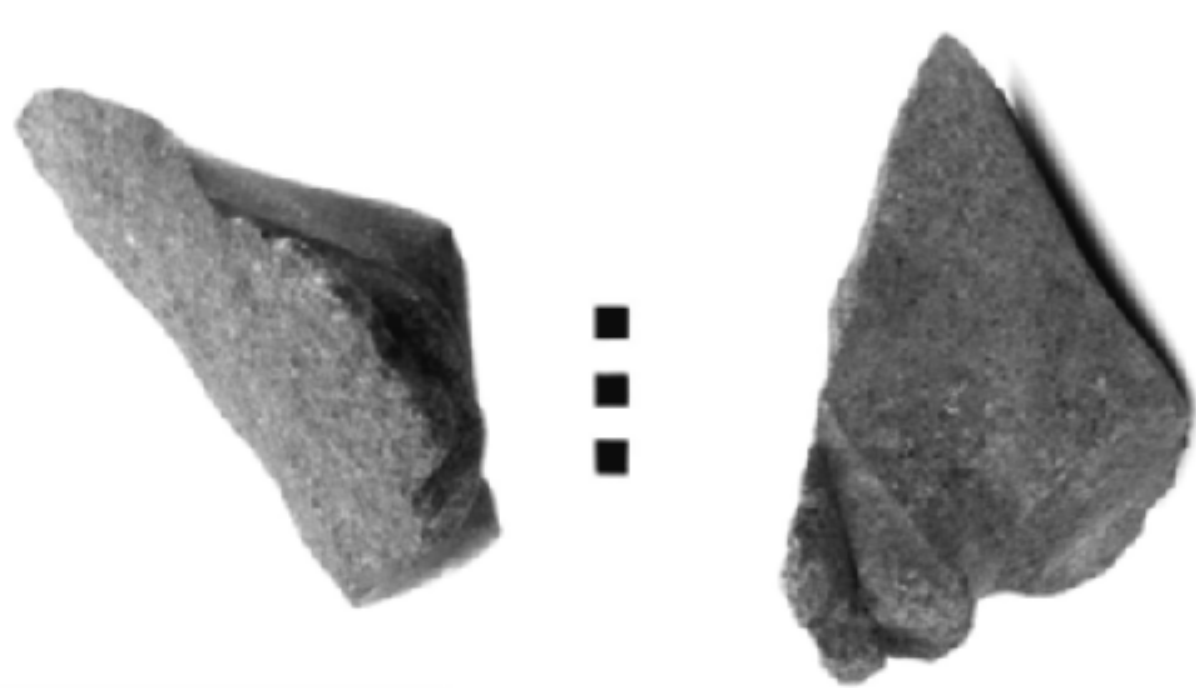

D. Laboury, Colosses et perspective. De la prise en considération de la statuaire pharaonique de grandes dimensions au Nouvel Empire. 


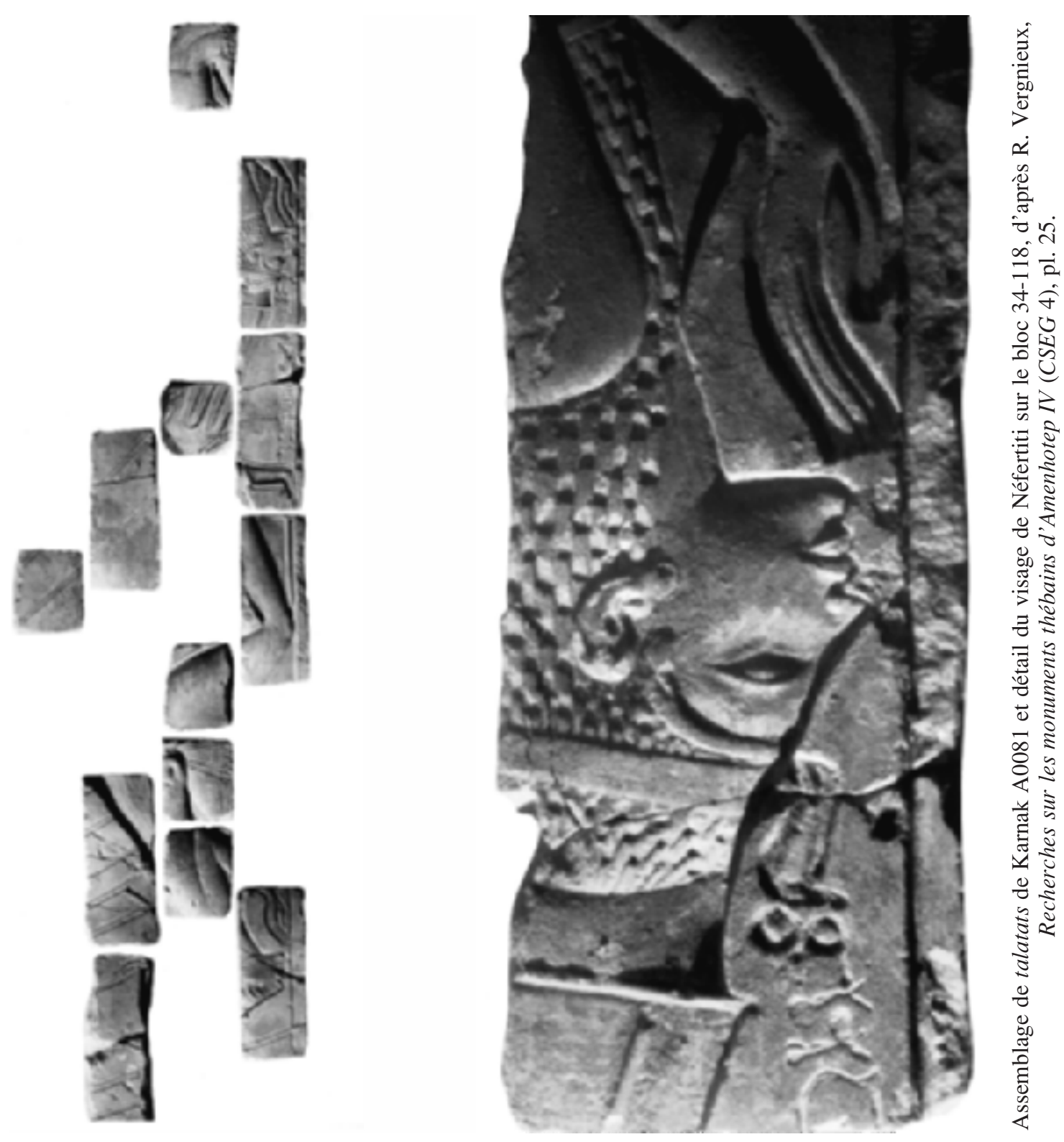

D. Laboury, Colosses et perspective. De la prise en considération de la statuaire pharaonique de grandes dimensions au Nouvel Empire. 


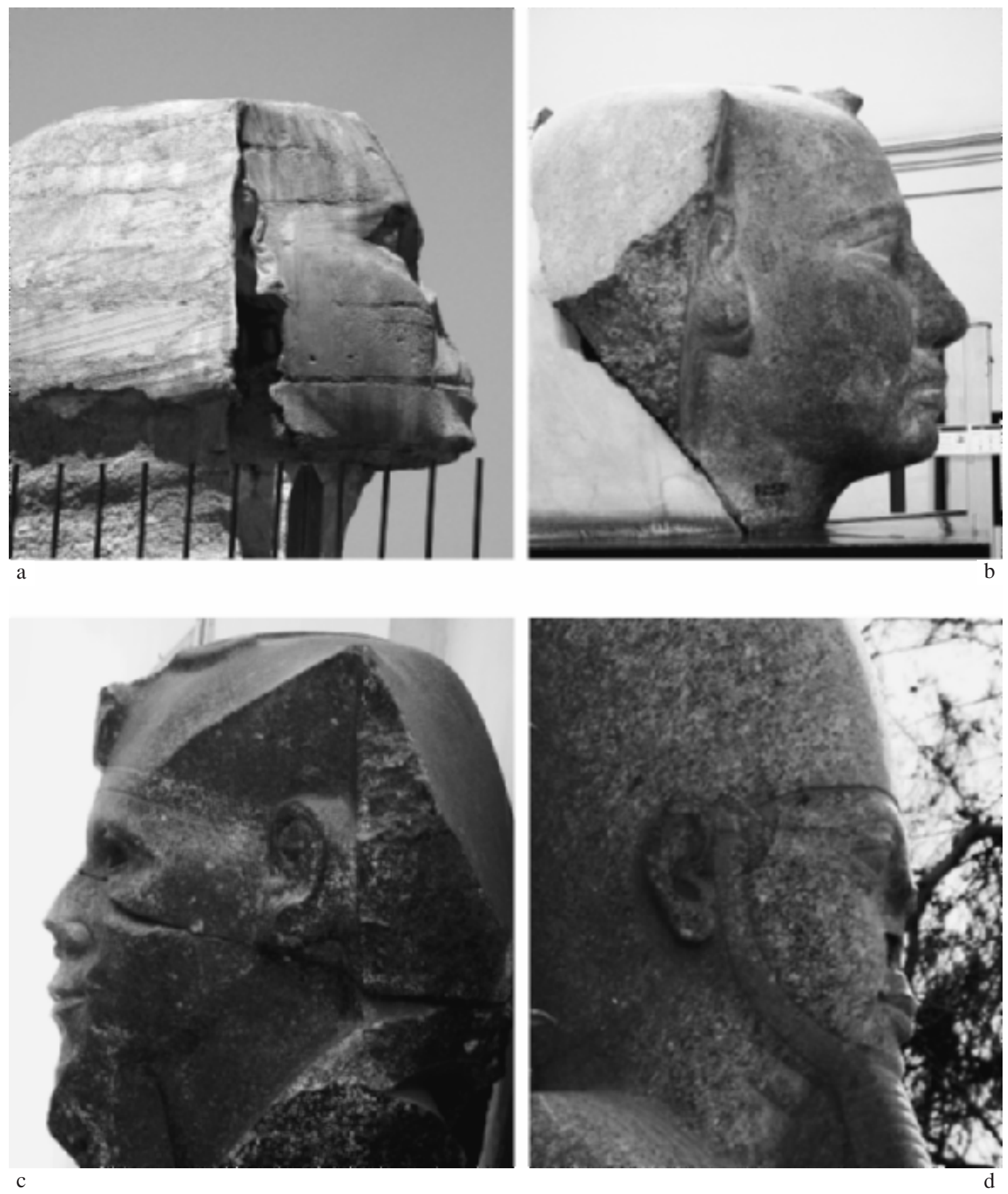

Vue de profil du grand sphinx de Guiza (a), des têtes Le Caire JE 52501 (b) et CG 383 (c), et d'un colosse de Sésostris I ${ }^{\text {er }}$ usurpé par Ramsès II à Mit Rahinah (d) (clichés de l'auteur).

D. Laboury, Colosses et perspective. De la prise en considération de la statuaire pharaonique de grandes dimensions au Nouvel Empire. 


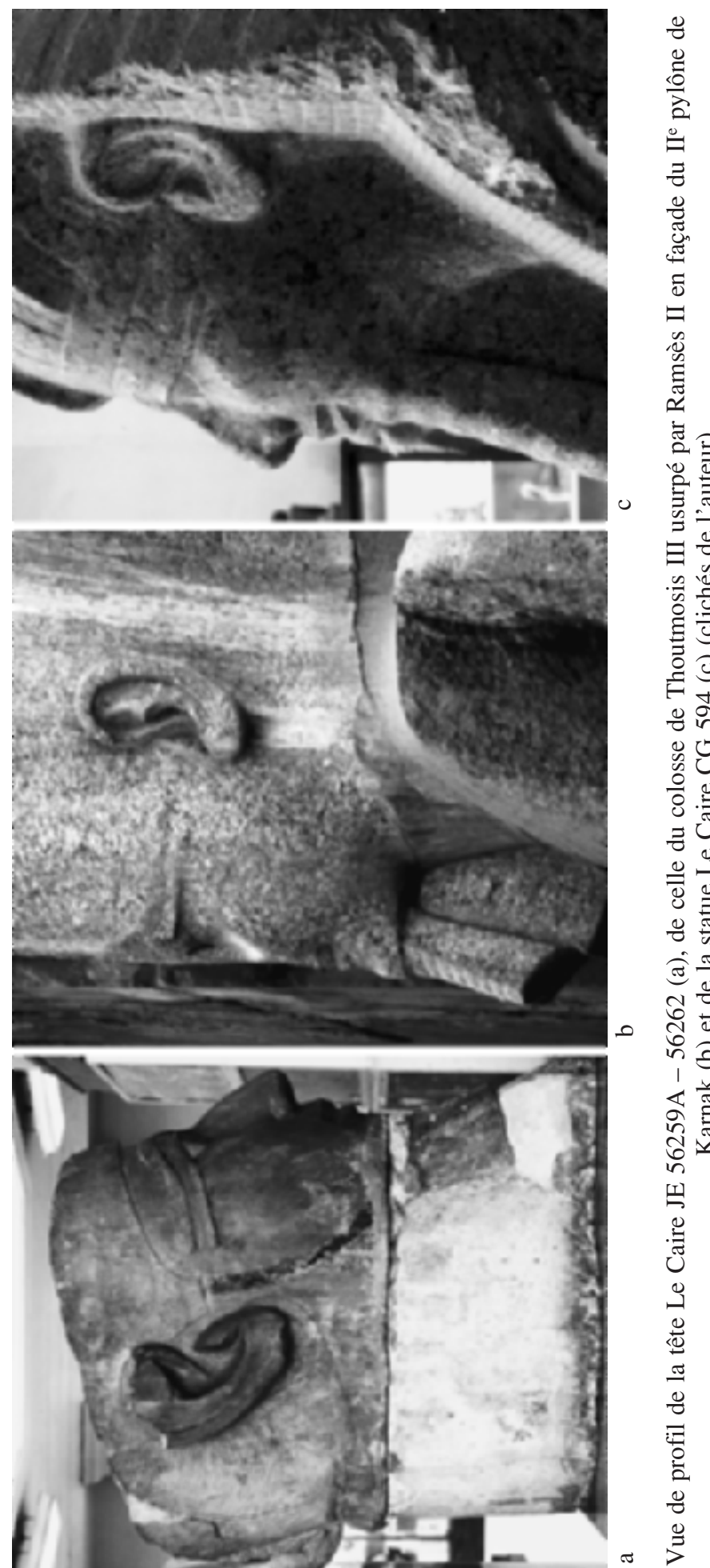

D. Laboury, Colosses et perspective. De la prise en considération de la statuaire pharaonique de grandes dimensions au Nouvel Empire. 


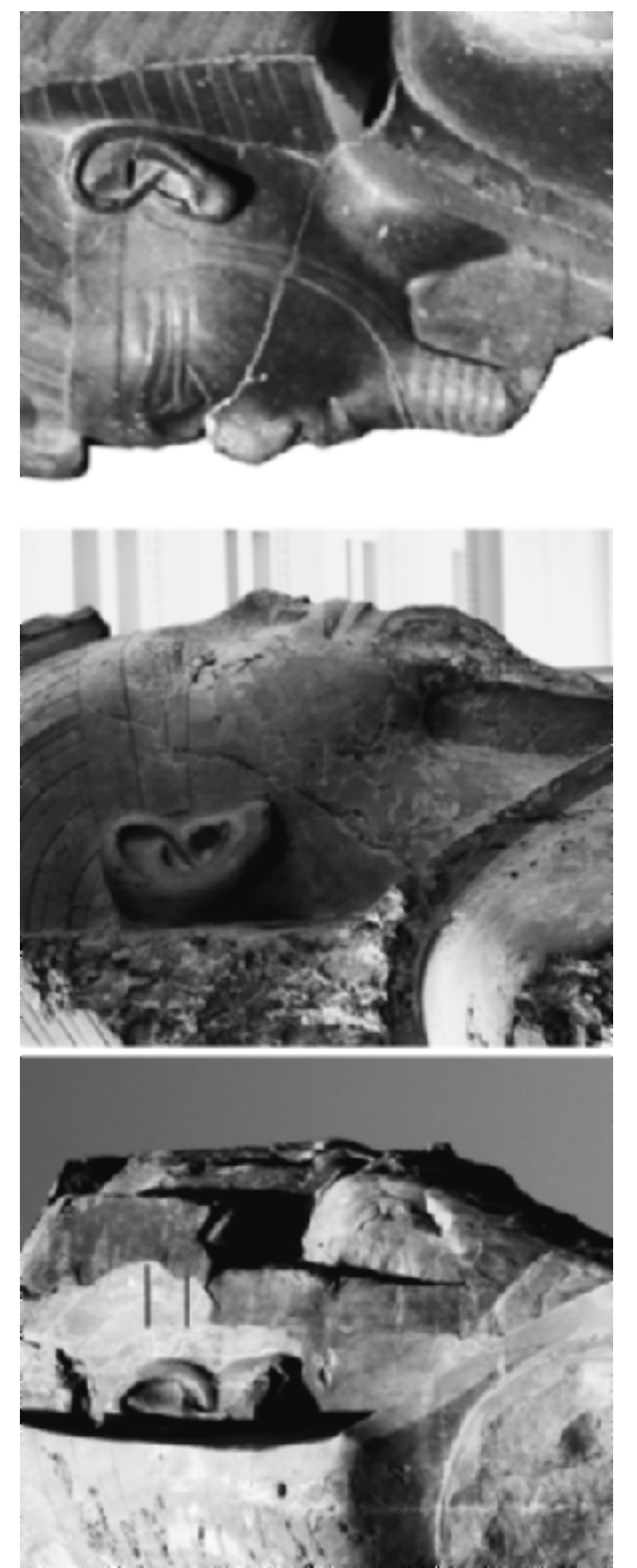

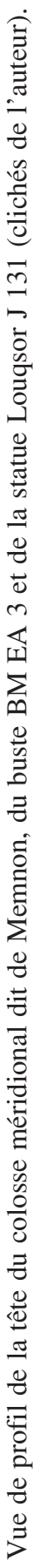

D. Laboury, Colosses et perspective. De la prise en considération de la statuaire pharaonique de grandes dimensions au Nouvel Empire. 


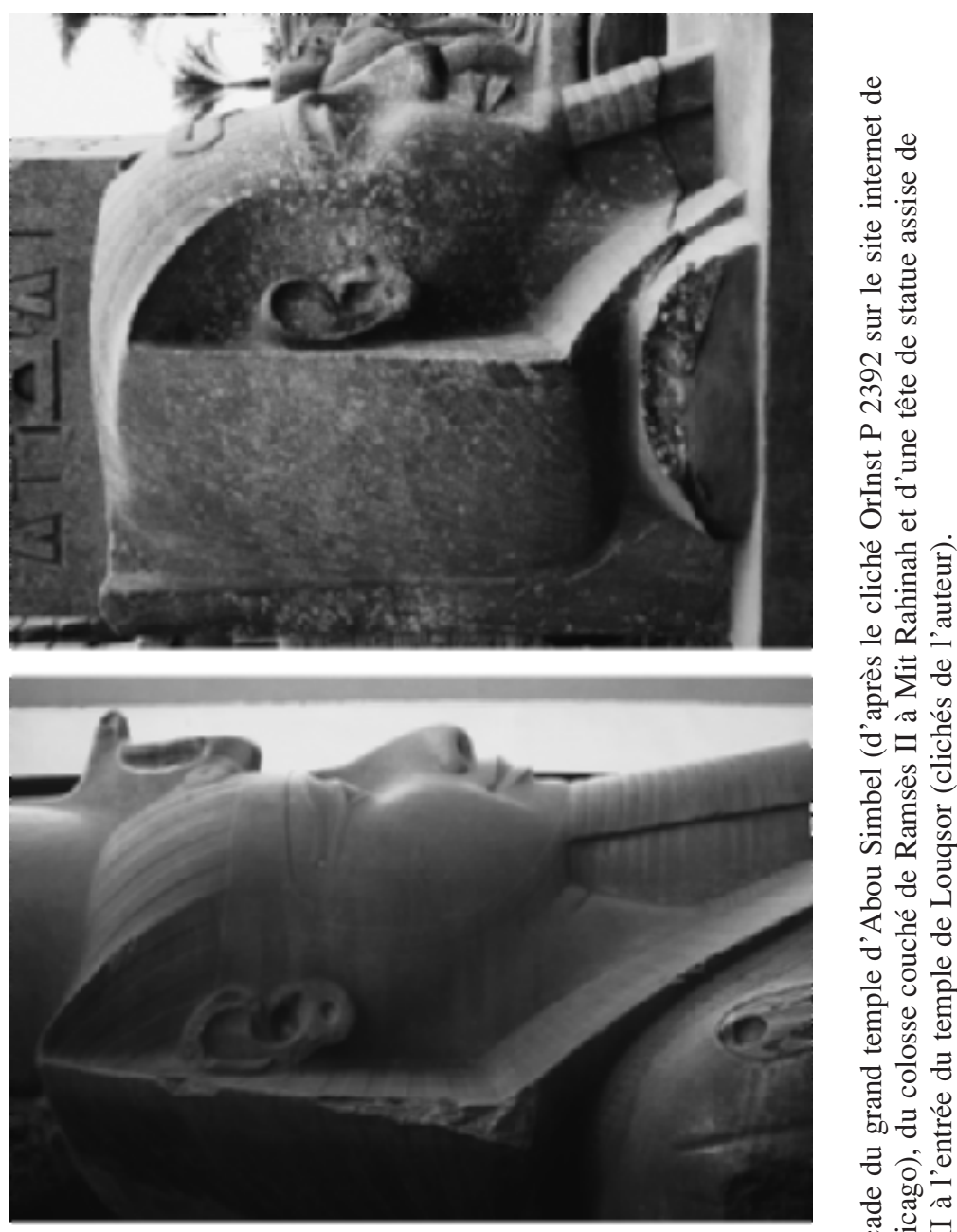

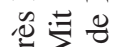

है,

르

造

है है

효

$<0$

$\because 0$

을

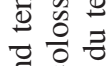

要过

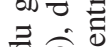

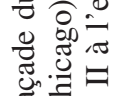

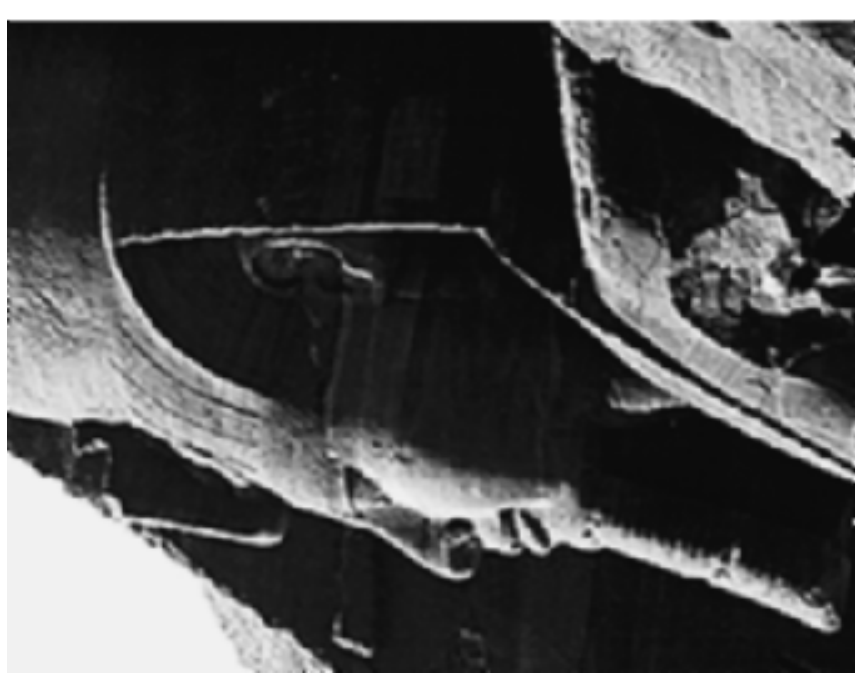

过出

워

플

을

营:

0

总

至气

믕 쥼

은

$\stackrel{0}{5}$

D. Laboury, Colosses et perspective. De la prise en considération de la statuaire pharaonique de grandes dimensions au Nouvel Empire. 


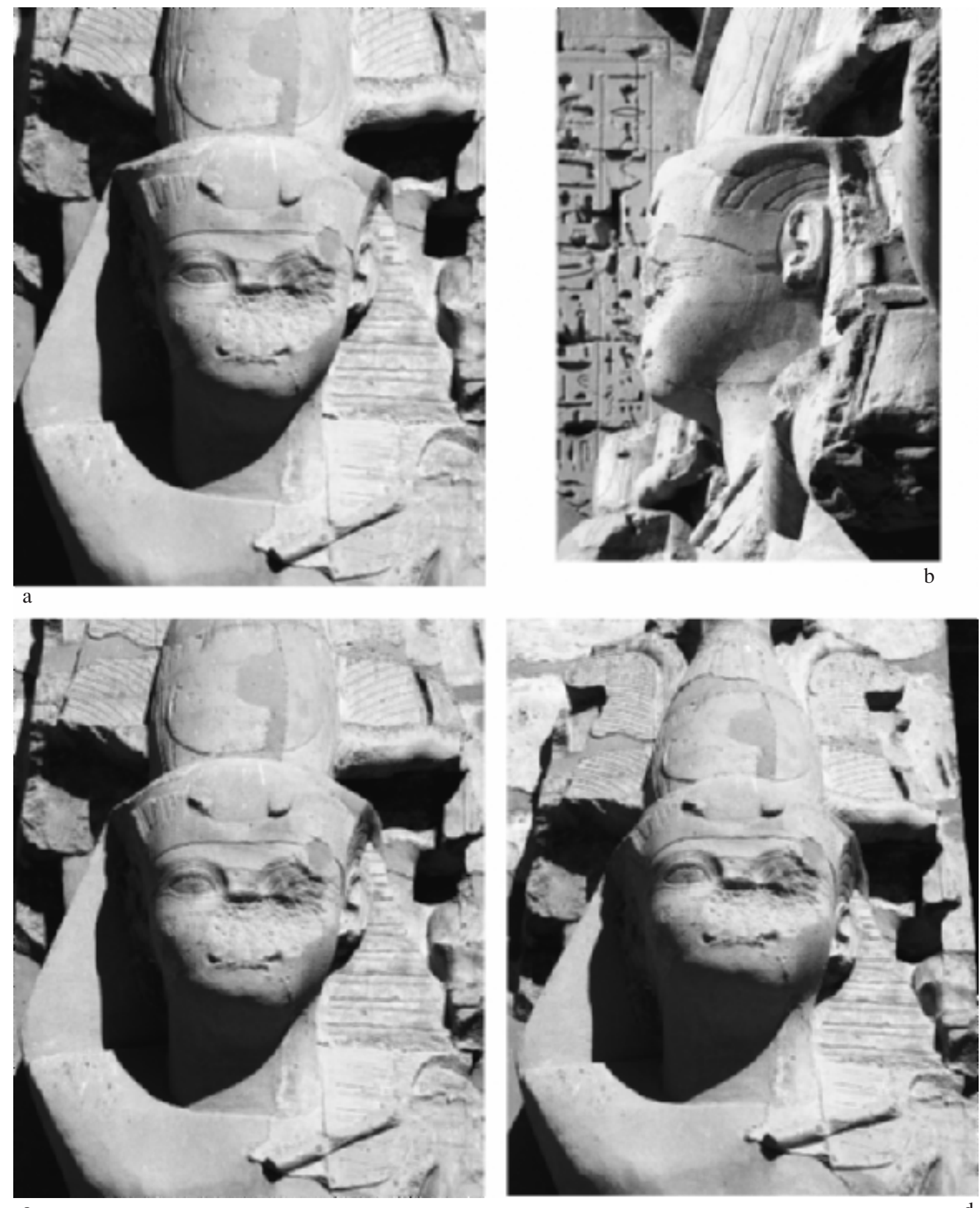

Vues de la tête d'un colosse osiriaque de la première cour du temple de Ramsès III à Médinet Habou, de face depuis l'extrémité sud de la cour (a), de profil (b), de face depuis l'axe central de la cour (c) et de face depuis le milieu de la moitié nord de la cour (d) (clichés de l'auteur).

D. Laboury, Colosses et perspective. De la prise en considération de la statuaire pharaonique de grandes dimensions au Nouvel Empire. 


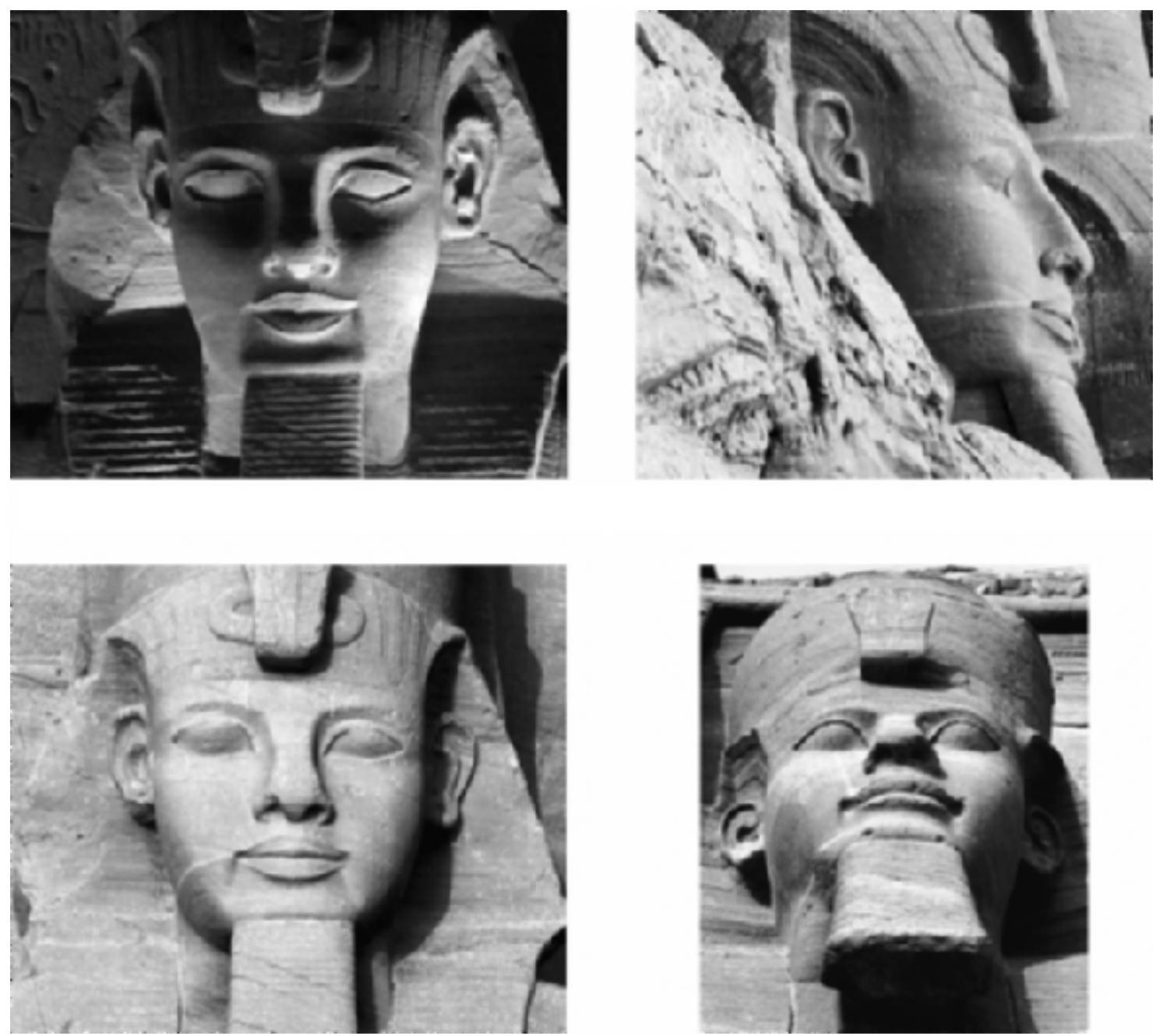

Vues de la tête d'un colosse assis en façade du grand temple d'Abou Simbel depuis la plate-forme moderne du son et lumière, de profil, depuis l'extrémité de l'esplanade antique menant au temple et depuis le pied d'un colosse jumeau (clichés de l'auteur).

D. Laboury, Colosses et perspective. De la prise en considération de la statuaire pharaonique de grandes dimensions au Nouvel Empire. 


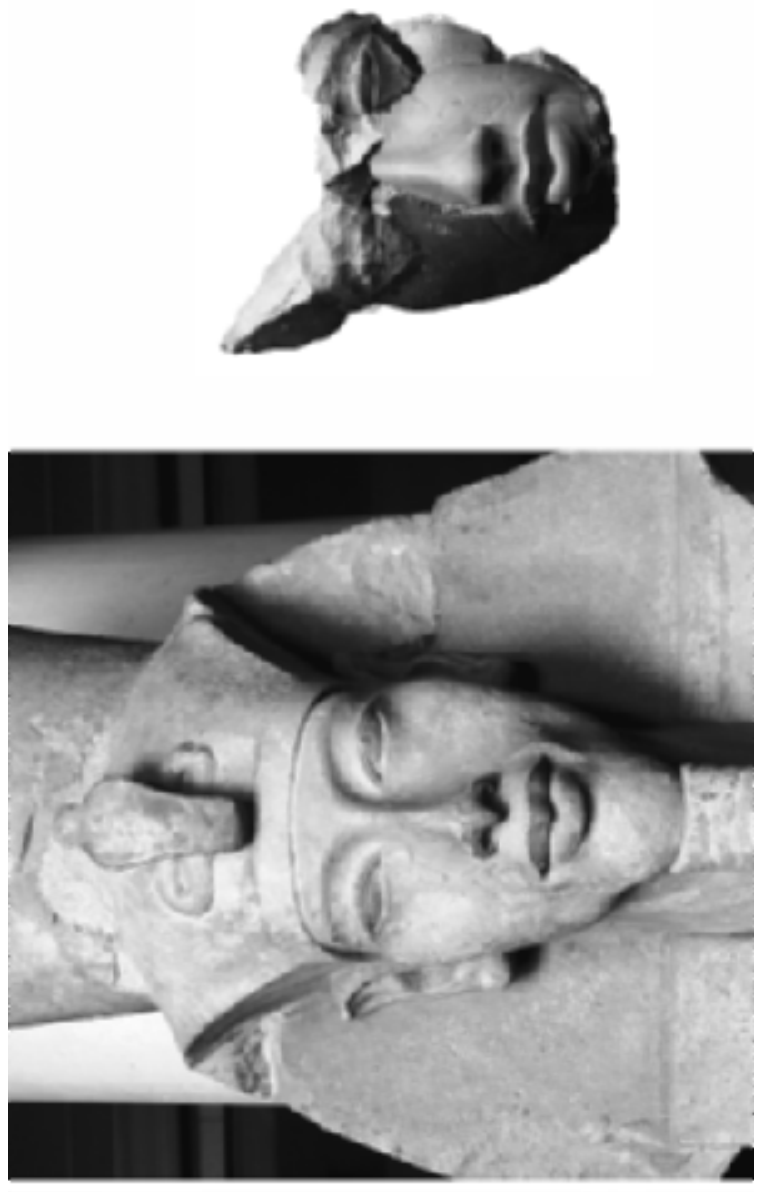

퐇ํำ

\&

ส

ํํ요

菨

过

वृ छे

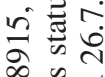

$\infty$ \&

더용

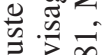

0

$\exists \underset{0}{0}$

氙 in

D。

으요

局 $\stackrel{0}{\circ}$

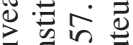

ป

ส․ํํำ

ه ते

实范

해워

政

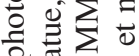

政象

实可 苞

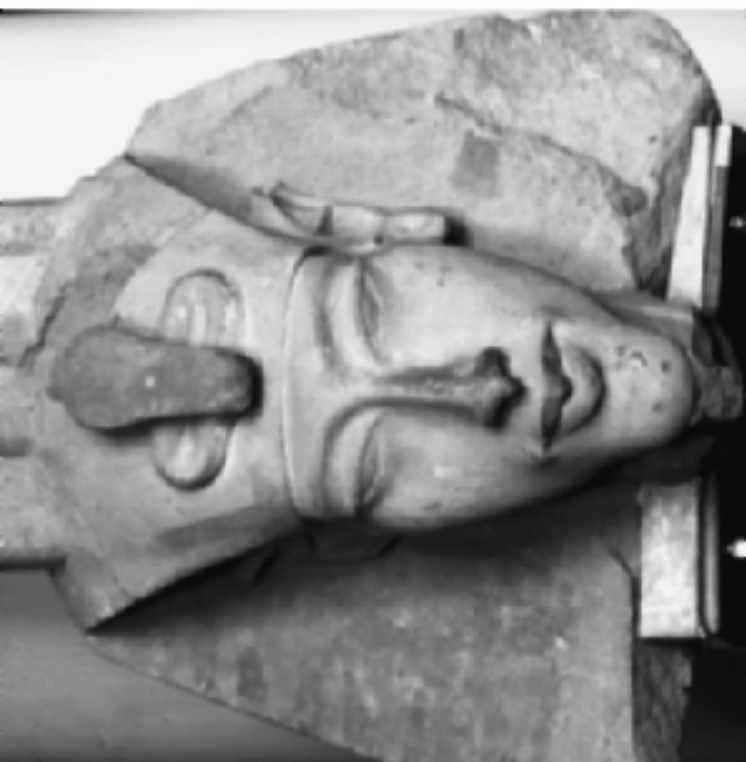

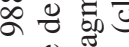

데용

o

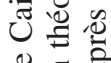

施

$\Phi$ 등

可.

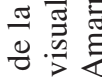

$0 \% 1$

赵. 言

o

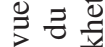

柾

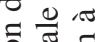

들

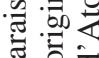

응

记离䓂

D. Laboury, Colosses et perspective. De la prise en considération de la statuaire pharaonique de grandes dimensions au Nouvel Empire. 